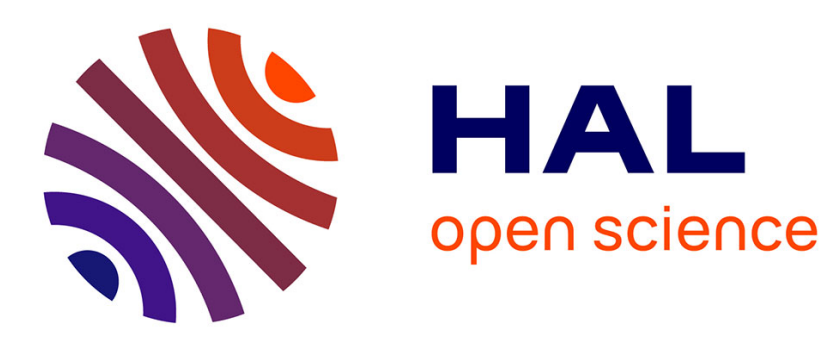

\title{
Une optimisation en temps de calcul pour la prédiction de zones de couverture radioélectriques
}

Pierre Combeau, Rodolphe Vauzelle, Lilian Aveneau, Yannis Pousset

\section{To cite this version:}

Pierre Combeau, Rodolphe Vauzelle, Lilian Aveneau, Yannis Pousset. Une optimisation en temps de calcul pour la prédiction de zones de couverture radioélectriques. Annals of Telecommunications annales des télécommunications, 2003, 58 (7-8), pp.1130-1156. 10.1007/BF03001874 . hal-00348488

\section{HAL Id: hal-00348488 \\ https://hal.science/hal-00348488}

Submitted on 26 Oct 2012

HAL is a multi-disciplinary open access archive for the deposit and dissemination of scientific research documents, whether they are published or not. The documents may come from teaching and research institutions in France or abroad, or from public or private research centers.
L'archive ouverte pluridisciplinaire HAL, est destinée au dépôt et à la diffusion de documents scientifiques de niveau recherche, publiés ou non, émanant des établissements d'enseignement et de recherche français ou étrangers, des laboratoires publics ou privés. 


\title{
Une optimisation en temps de calcul pour la prédiction de zones de couverture radioélectriques
}

A computation time optimization for the radio coverage prediction.

\author{
Pierre Combeau, Rodolphe Vauzelle, Lilian Aveneau, Yannis Pousset \\ Université de Poitiers \\ IRCOM-SIC UMR CNRS n 6615 \\ Bâtiment SP2MI, Téléport 2, boulevard Marie et Pierre Curie \\ BP 3017986962 FUTUROSCOPE CHASSENEUIL Cedex, France. \\ Tél : 0549496567 \\ Fax : 0549496570 \\ e-mail : combeau@sic.sp2mi.univ-poitiers.fr
}




\title{
Résumé
}

Cet article présente une méthode d'optimisation du temps de calcul pour la prédiction d'une zone de couverture radioélectrique, quel que soit le modèle de propagation utilisé. Le principe consiste à réduire le nombre de points d'application du modèle de propagation par rapport à une technique classique. La méthode proposée est basée sur une analyse multirésolution de signaux mesurés à environ $2 \mathrm{GHz}$, et sur une analyse électromagnétique de l'environnement de propagation. Les performances de la méthode sont évaluées en termes de réduction du temps de calcul et de précision, en comparaison avec la technique classique.

\begin{abstract}
This article presents a method which optimises the computation time for the prediction of a radio coverage, whatever the propagation model used. The principle consists in reducing the number of application points of the propagation model in relation to a classical technique. The proposed method is based on a multi resolution analysis of measured signals carried out around $2 \mathrm{GHz}$, and on an electromagnetic analysis of the propagation environment. The performances of the method are evaluated in term of reduction in computation time and of accuracy, in comparaison with the classical technique.
\end{abstract}

\section{Introduction}

Ces dernières années ont connu un développement rapide et considérable du marché des radiocommunications mobiles avec les systèmes de deuxième génération $(2 \mathrm{G})$ et bientôt avec la mise en place de la 3G (UMTS). Un nombre d'utilisateurs sans cesse croissant a rendu urgent la nécessité d'assurer une couverture radioélectrique de bonne qualité. Lors du déploiement de tels réseaux, il a fallu mettre au point des modèles performants, capables de prédire la zone de couverture radioélectrique des différents émetteurs placés en des points stratégiques [(W.93].

Rappelons la définition de la zone de couverture radioélectrique d'un émetteur donné : il s'agit de la zone géographique dans laquelle les signaux radioélectriques reçus de cet émetteur ont une amplitude moyenne supérieure à un seuil lié à une qualité de communication acceptable [(L.83].

Les techniques classiques de calcul de telles zones utilisent un modèle de propagation d'ondes électromagnétiques, qui est soit vectoriel [GA00, FYLR01] soit scalaire [(J.91]. Ce modèle est appliqué sur un ensemble de points de réception fictifs, répartis selon un pas spatial constant sur la zone géographique étudiée. Ce pas est généralement de quelques mètres en milieu urbain [(X.00]. Cette méthode conduit à des temps de calcul très importants voire prohibitifs dans des environnements géographiques complexes [(C.90]. Des approches existent pour réduire les temps de calcul de cette prédiction; elles consistent à simplifier la complexité du modèle de propagation utilisé [(M.80, (Y.68, LYRM00].

Notre travail s'inscrit dans une démarche différente et complémentaire des approches précédentes. Elle est différente, car elle vise à réduire le nombre de points d'application du modèle de propagation, plutôt que la complexité de ce dernier. Elle est donc complémentaire, car indépendante des modèles de propagation et de leurs optimisations.

La méthode proposée dans cet article s'appuie sur l'hypothèse suivante : le signal reçu par un mobile présente des variations induites par divers phénomènes électromagnétiques de propagation des ondes. Chaque type de variation est alors dû à une combinaison particulière de ces phénomènes. En étendant ce principe à une zone géographique, celle-ci peut être considérée comme une partition spatiale dont chaque élément se caractérise par une variation homogène des signaux reçus, i.e par une combinaison identique d'interactions électromagnétiques subies par les ondes.

Partant de cette hypothèse, exposée dans le paragraphe 1, nous proposons de réduire le temps de calcul nécessaire à la prédiction d'une zone de couverture en limitant l'application d'un modèle de propagation à seulement quelques points dans chaque élément d'une telle partition. La vérification de cette hypothèse s'appuie sur deux outils présentés et mis en oeuvre dans les paragraphes 2 et 3 . Le premier est un logiciel d'analyse électromagnétique qui permet d'identifier les différentes régions de la zone géographique étudiée présentant un mécanisme particulier de propagation subi par les ondes. Le deuxième outil est un logiciel de segmentation multiéchelle pour la détection des changements de comportement des signaux reçus par un récepteur mobile. L'application de ces techniques est ensuite présentée pour la vérification de l'hypothèse formulée. 
Le paragraphe 4 présente notre méthode optimisée de prédiction de la zone de couverture d'un émetteur radiomobile. Nous justifions en particulier la réduction du nombre de points d'application du modèle par élément.

L'évaluation des performances de notre méthode en termes de temps de calcul et de précision fait l'objet du paragraphe 5. Deux configurations sont considérées, à savoir petite cellule et micro cellule.

\section{Hypothèse}

Dans le contexte des radiocommunications mobiles, entre un émetteur et un récepteur, l'information se propage par trajets multiples comme le montre la figure 19; le signal reçu en un point résulte ainsi de la combinaison d'ondes ayant suivi des chemins différents.

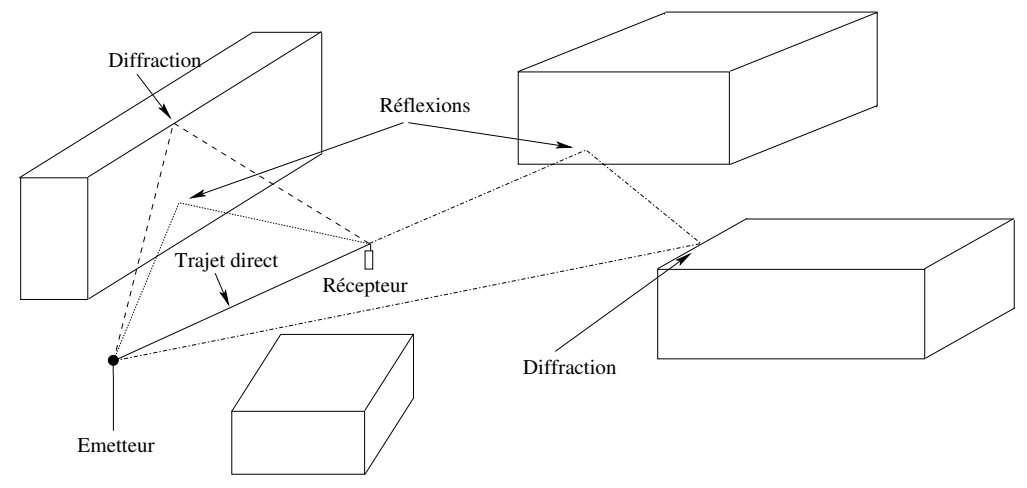

figure 1 - Propagation par trajets multiples (E : émetteur, $\mathrm{R}$ : récepteur)

Au cours de sa propagation, chaque onde peut subir une combinaison particulière d'interactions électromagnétiques avec l'environnement de propagation [(J.92]. Classiquement, une interaction électromagnétique est définie comme étant soit le phénomène de réflexion sur des surfaces, soit le phénomène de diffraction sur des arêtes d'objets rencontrés par les ondes. Dans le cas où l'onde ne subit aucune interaction, elle se propage alors en visibilité directe. En reprenant l'exemple de la figure 19, on constate que le signal reçu est dû à une onde directe, une onde réfléchie, une onde diffractée, une onde diffractée puis réfléchie, ....

Sur la base des quelques notions de propagation très brièvement rappelées et de notre observation de nombreux signaux radioélectriques acquis lors de campagnes de mesures [YRL03], nous formulons l'hypothèse suivante afin de pouvoir réduire le temps nécessaire à la prédiction d'une zone de couverture.

Hypothèse : Les variations d'un signal radioélectrique, reçu par un récepteur mobile sur un parcours, sont homogènes pour une même combinaison d'interactions électromagnétiques (l'onde en visibilité, lorsqu'elle existe, doit y être intégrée).

En étendant cette hypothèse à une zone géographique, on peut supposer que l'ensemble des points pour lesquels le signal reçu résulte d'une même combinaison d'interactions électromagnétiques conduit à délimiter une région de l'environnement dans laquelle la dynamique et le niveau moyen du signal varient peu. Nous en déduisons alors que la zone géographique étudiée est une partition spatiale composée de différents éléments dans lesquels le signal reçu a un comportement homogène. Passer d'un élément à un autre revient donc à considérer des signaux dont la "forme" change. La figure 20 permet d'illustrer cette hypothèse sur un exemple schématique.

En considérant la position de l'émetteur par rapport au bâtiment comme l'illustre la figure 20 (a), l'application des lois physiques associées à la propagation des ondes [(X.00] fait apparaitre différentes régions caractérisées chacune par une combinaison d'interactions électromagnétiques : (1) simple diffraction, (2) visibilité et (3) visibilité plus réflexion. Un récepteur mobile, effectuant le parcours schématisé sur la figure 20 (a), va traverser différentes régions de la partition et ainsi recevoir, selon l'hypothèse formulée, le signal schématique de la figure 20 (b). En effet, à chaque région est associée une combinaison particulière d'interactions électromagnétiques induisant un comportement spécifique du signal.

Les points $P_{1 a}, P_{2 a}, P_{3 a}$ et $P_{4 a}$ correspondant aux changements de régions traversées par le parcours sont donc fortement corrélés aux points $P_{1 s}, P_{2 s}, P_{3 s}$ et $P_{4 s}$, indiquant des modifications significatives de la forme du signal reçu.

Compte tenu de cette hypothèse, pour optimiser le temps de calcul de la zone de couverture, il de- 


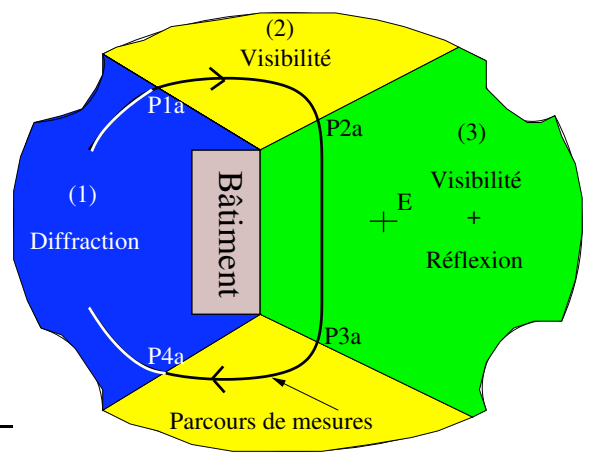

(a)

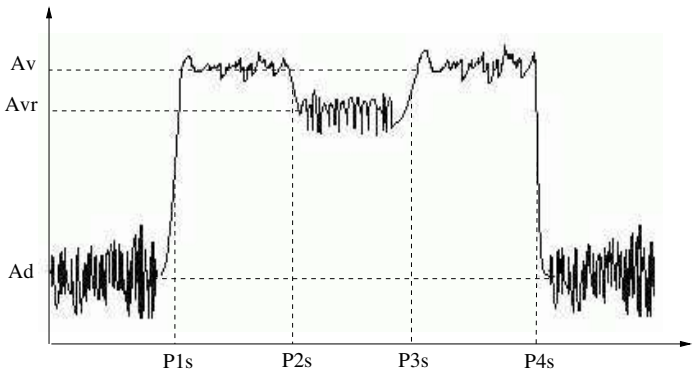

(b)

figure 2 - (a) Partition spatiale (b) Signal schématique reçu sur le parcours de mesure

vient possible d'appliquer un modèle de propagation sur seulement quelques points de chaque élément, puisque le niveau moyen varie peu, et d'extrapoler le résultat à l'élément tout entier. Le modèle n'est alors plus appliqué selon un pas spatial constant, contrairement à la technique classique.

Le coeur de la méthode proposée est constitué d'un outil d'analyse électromagnétique de l'environnement. Il permet de décomposer le milieu en régions caractérisées par des combinaisons particulières de phénomènes physiques. Plus précisément, cette analyse est appliquée lors de la phase de vérification de l'hypothèse (paragraphe 3 ) et lors de la mise en oeuvre de la méthode (paragraphe 4).

Toutefois, un autre outil contribue à la vérification de l'hypothèse; il s'agit d'une technique d'analyse des signaux acquis sur des parcours prédéfinis, permettant de détecter leurs ruptures significatives.

\section{Analyse électromagnétique de l'environnement}

L'analyse électromagnétique de l'environnement de propagation a pour objectif de produire une partition spatiale de cet environnement. Chaque élément de cette partition est caractérisé par une même combinaison d'interactions (diffraction, réflexion et également l'onde directe). Toutefois, bien que la propagation des ondes soit un phénomène tridimensionnel, le logiciel d'analyse électromagnétique n'a pas été réalisé en 3D compte tenu de la difficulté de cette tâche. Il a été choisi de tendre progressivement vers cette solution, théoriquement idéale, à travers plusieurs versions de complexité croissante. Initialement, une version produisant une partition dans un plan horizontal a été développée, puis une version reposant sur une étude dans des plans verticaux et enfin une version effectuant une étude de la propagation en 2,5D. Son résultat correspond à une fusion des partitions précédentes. Néanmoins, quelle que soit la version considérée, la partition est toujours calculée à une hauteur de 1,5 m au-dessus du sol ; cette hauteur est celle retenue généralement comme étant la hauteur moyenne d'un récepteur mobile.

\subsection{Analyse électromagnétique horizontale (2DH)}

D'une façon générale, le logiciel d'analyse électromagnétique repose sur la recherche des frontières optiques créées par les faces réfléchissantes et les arêtes diffractantes des éléments qui constituent l'environnement. Dans le cas d'une analyse dans un plan horizontal, les phénomènes de réflexion et de diffraction produisent les effets illustrés respectivement par les figures 3 (a) et (b).

Le bâtiment engendre des zones de réflexion lorsque ses faces sont en visibilité directe de l'émetteur. Elles sont définies en respectant les lois de l'optique géométrique qui indiquent que l'angle de réflexion est égal à l'angle d'incidence. De même, le bâtiment engendre deux zones de diffraction sur les coins notés (1) et (2), comme l'illustre la figure 21 (b). Pour déterminer les zones diffractées par les arêtes d'un bâtiment, il est nécessaire de réaliser une étude préalable. Théoriquement, les ondes diffractées occupent tout l'espace laissé libre par l'obstacle [(J.62, RP74]. Toutefois, l'intensité des ondes diffractées diminue lorsque l'angle $\alpha$ augmente (figure 22). Sur ce constat, on définit un angle $\alpha_{l}$ comme étant la limite perceptible de l'influence des ondes diffractées par rapport à l'onde directe. Pour un angle supérieur à $\alpha_{l}$, les ondes diffractées sont trop atténuées et peuvent être négligées.

Il a été montré [YRL03] que l'angle $\alpha$ est toujours inférieur à environ 3 degrés pour des fréquences supérieures au gigahertz et ceci quelles que soient les situations (distance, angle d'ouverture des dièdres 


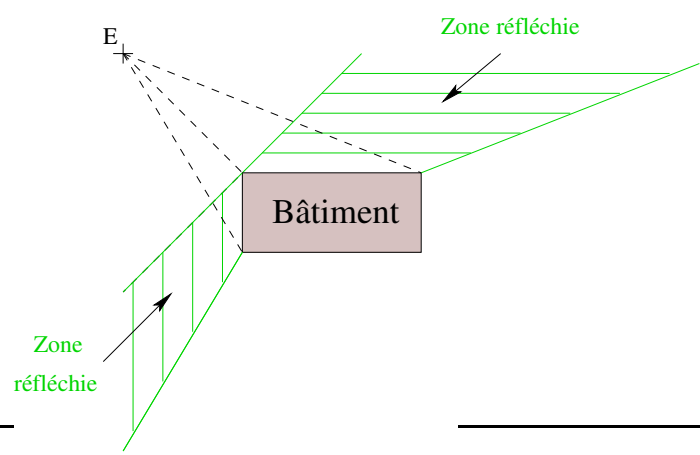

(a)

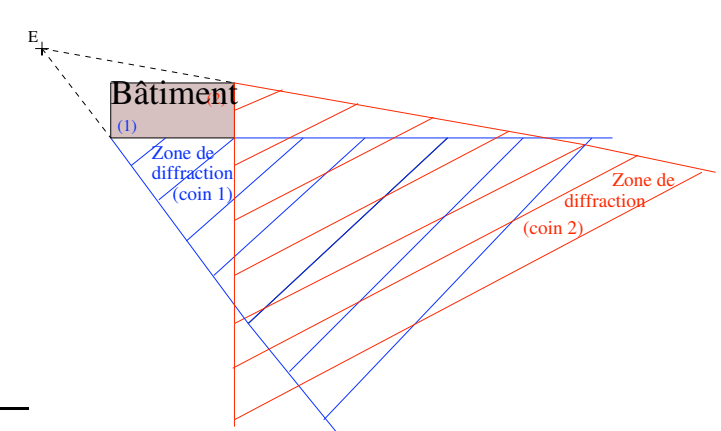

(b)

figure 3 - Zones réfléchies (a) et diffractées (b) par un bâtiment

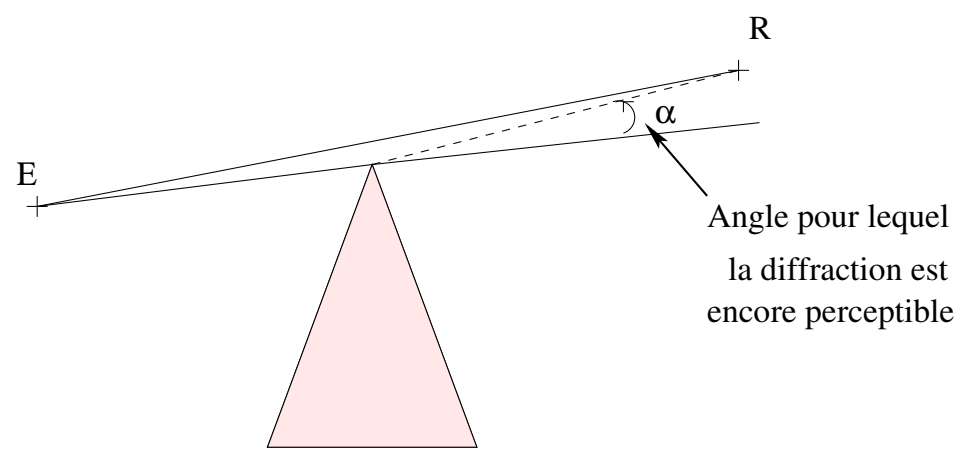

figure 4 - Limite de l'influence des ondes diffractées liée à $\alpha$

et nature des dièdres). Cet angle est très faible par rapport aux grandeurs mises en jeu dans les environnements étudiés et peut donc être considéré comme nul. Dans l'analyse électromagnétique, la diffraction n'est donc prise en compte que dans les zones d'ombre des obstacles.

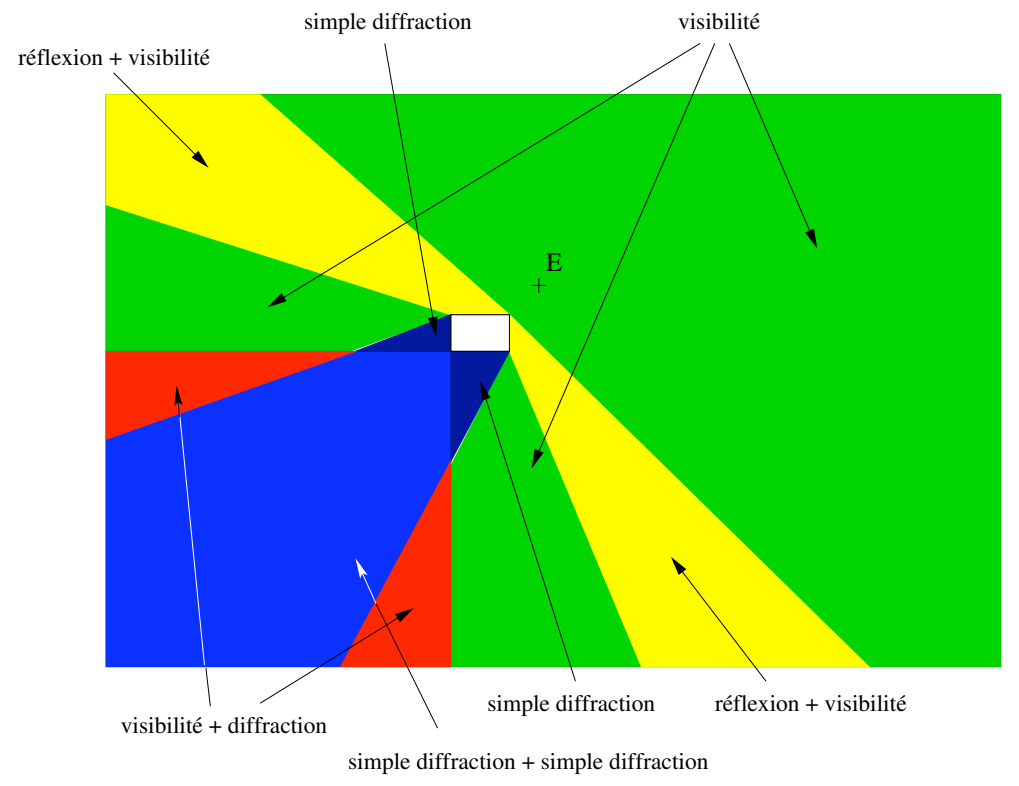

figure 5 - Les différentes zones engendrées par un bâtiment pour une diffraction et une réflexion

On peut observer sur la figure 21 (b) la superposition partielle de deux zones de diffraction. En effectuant un parallèle avec une approche de type rayons, cela signifie qu'un récepteur situé dans cette zone de superposition recevrait deux trajets simplement diffractés.

Dans le cas d'un bâtiment, en associant les phénomènes précédents de réflexion et de diffraction, 
l'analyse électromagnétique fournit le résultat proposé sur la figure 23. Il s'agit d'une partition spatiale composée de différents éléments dont les niveaux de gris permettent d'identifier la nature des interactions associées. A nouveau, il faut souligner l'existence d'éléments définis par des superpositions de zones initiales tels que l'élément correspondant à l'intersection d'une zone en visibilité directe et d'une zone de simple réflexion.

Compte tenu de l'objectif de l'analyse électromagnétique qui est de fournir une partition pour un calcul de couverture, c'est à dire ne considérant que des variations lentes significatives des signaux reçus, la partition finale ne doit être constituée que des éléments traduisant de telles variations. En s'appuyant sur une hiérarchisation des phénomènes physiques selon leur atténuation engendrée sur les ondes [YRL03], l'élément caractérisé par une visibilité directe et une réflexion est finalement considéré comme un élément en visibilité. En effet, il correspond à des points recevant un trajet direct plus énergétique que le trajet réfléchi. Sur de telles considérations et après d'éventuels regroupements de zones contigües ayant des caractéristiques très voisines (c'est à dire caractérisées par des atténuations peu différentes), la partition finale de l'analyse électromagnétique est représentée sur la figure 24.

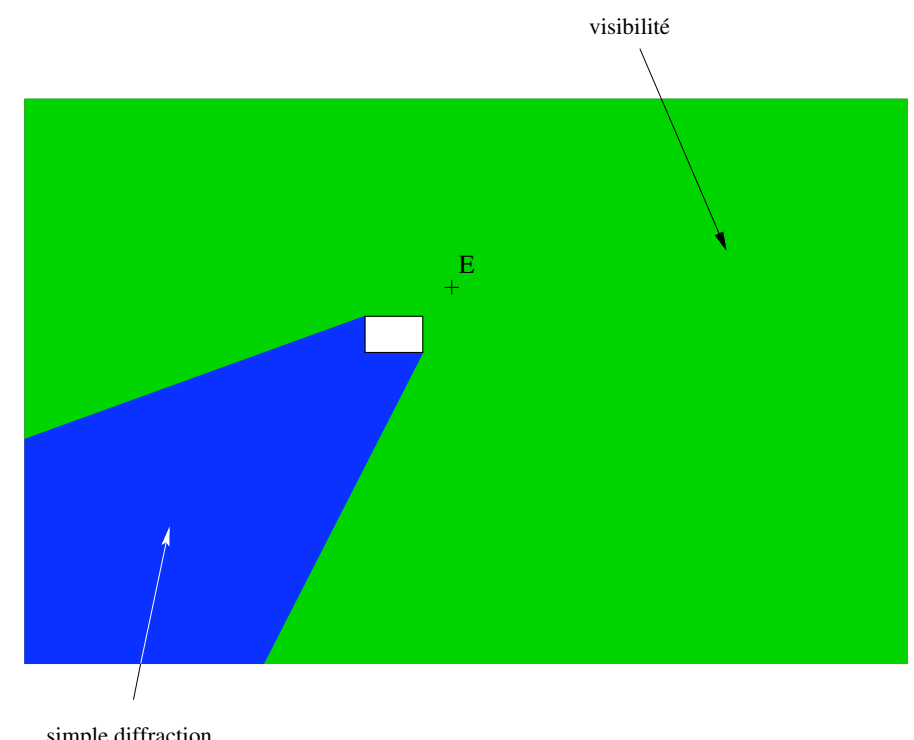

figure 6 - Résultat du regroupement

\subsection{Analyse électromagnétique verticale (2DV)}

L'analyse électromagnétique développée en vertical s'appuie sur la même démarche qu'en horizontal mais est légèrement plus lourde à mettre en oeuvre.

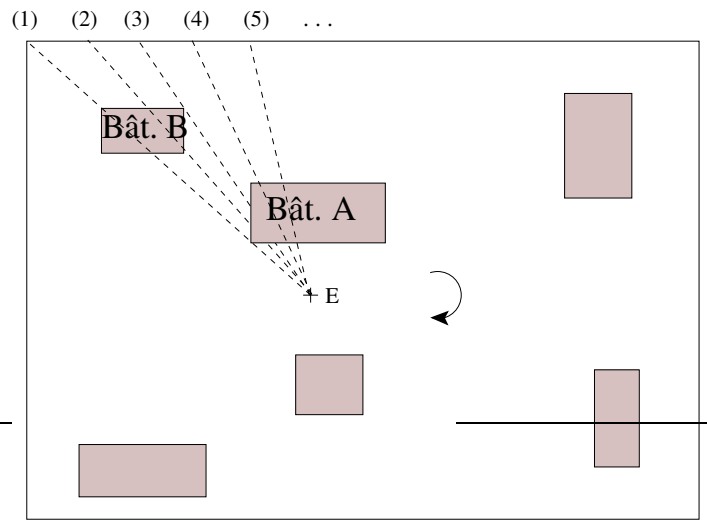

(a)

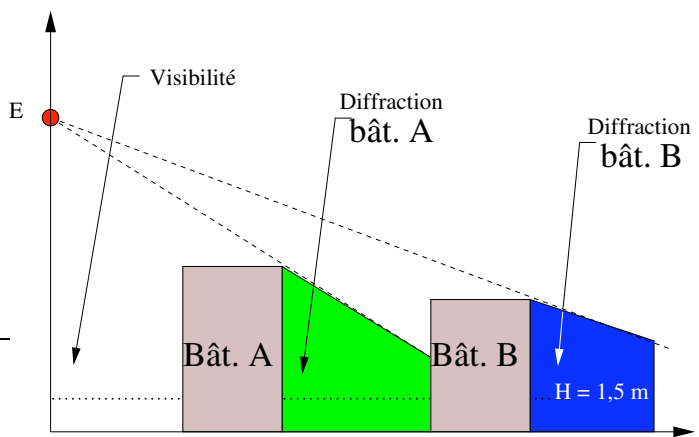

(b)

figure 7 - (a) Balayage régulier de l'environnement par des plans verticaux (b) Analyse électromagnétique du plan noté (2) pour 1 diffraction et 0 réflexion 
En effet, dans la version verticale, le résultat est toujours fourni dans un plan horizontal à 1,5 mètre au dessus du sol; par contre, l'analyse de la propagation se fait dans une succession de plans verticaux. La figure 25 (a) montre un exemple schématique constitué de bâtiments, traité avec une diffraction. L'information contenue dans chaque plan à 1,5 mètre du sol est ensuite extraite (figure 25 (b)) pour constituer une partition initiale dans le plan horizontal à cette hauteur (figure 26).

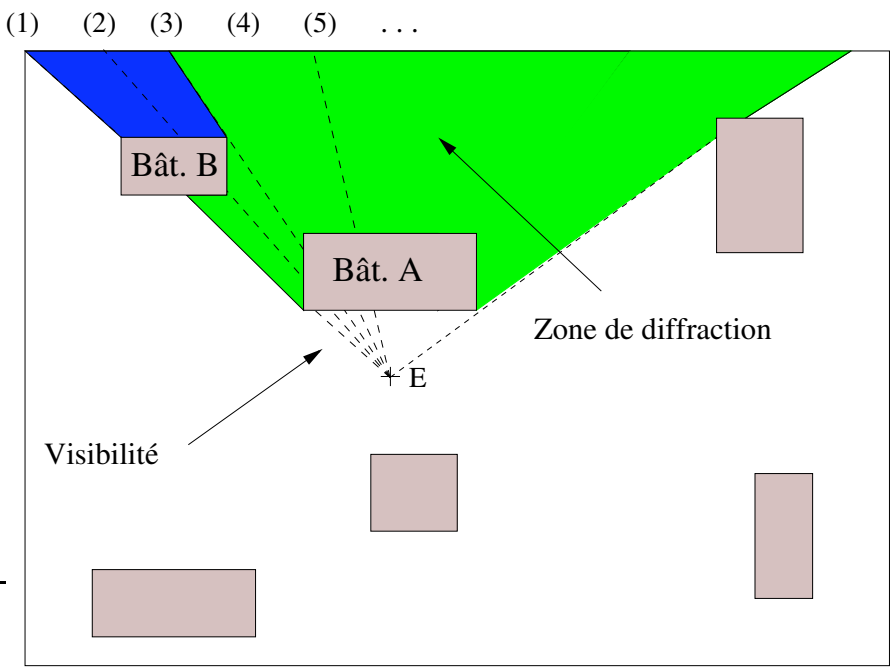

figure 8 - Construction d'une partition issue de l'analyse électromagnétique en verticale

Ensuite, le principe de hiérarchie des interactions et d'éventuels regroupements d'éléments contigüs est appliqué à cette partition, comme pour la version horizontale, afin de produire la partition finale.

\subsection{Analyse électromagnétique 2,5D}

Cette version consiste à associer les deux analyses précédentes (horizontale et verticale). A nouveau, le résultat est une partition présentée dans un plan horizontal à 1,5 mètre du sol.

Le calcul effectif de cette partition repose sur l'application du principe de hiérarchie des interactions et de regroupement sur une partition résultant de la fusion des partitions initiales obtenues en horizontal et en vertical.

\subsection{Paramètres de l'analyse électromagnétique}

Quelle que soit la version considérée, le logiciel d'analyse électromagnétique a comme informations d'entrée des données caractérisant l'environnement de propagation. Ce dernier est défini par des bases de données, fournissant en 3D le sol et le sursol, établies par l'Institut Géographique National. En outre, l'analyse est paramétrée par le nombre et la nature des interactions électromagnétiques - diffraction et réflexion - prises en compte pour le calcul de la partition.

Ces paramètres sont essentiels car ils interviennent directement sur la finesse de la partition. Considérer des combinaisons complexes d'interactions revient à déterminer une partition constituée d'un grand nombre d'éléments. Comme le montre la figure 20, il est estimé que les paramètres sont corrects lorsque le signal reçu sur un parcours présente des changements significatifs de comportement au voisinage des frontières des éléments de la partition spatiale.

Les valeurs de ces paramètres sont obtenues à l'issue de la vérification de l'hypothèse comme l'explique le paragraphe suivant.

\section{Vérification de l'hypothèse}

L'objet de ce paragraphe est de montrer la validité de notre hypothèse qui est la base de notre méthode d'optimisation pour la prédiction de zone de couverture. Cette hypothèse, rappelons le, conjecture l'existence d'une très forte corrélation entre les variations lentes et significatives de signaux mesurés sur le terrain et les mécanismes de propagation des ondes radioélectriques. Ainsi, nous tentons d'établir la correspondance entre les variations du signal mesuré et la partition spatiale obtenue par analyse 
électromagnétique. Dans ce but, nous avons mis en oeuvre une technique de segmentation de signaux permettant d'identifier leurs variations significatives, présentée au paragraphe suivant.

\subsection{Segmentation de signaux}

Notre objectif est de décomposer un signal en différents éléments. Les maxima d'ondelettes constituent un outil qui, par ses propriétés, permet d'atteindre ce but. En effet, l'analyse multiéchelle permet d'étudier un signal à différentes échelles [(S.88]. Chacune d'elles correspond à une bande de fréquences particulière; de plus à une montée en échelle correspond une descente en fréquences. Ainsi, sonder les basses fréquences du signal revient à le lisser. La fonction lissant le signal et permettant ainsi de calculer l'échelle suivante est appelée fonction d'échelle.

Les variations rapides, perdues lors du lissage lors d'un changement d'échelle, sont projetées dans la base d'ondelettes complémentaires [(P.93].

Si l'ondelette $\psi^{1}(t)$ est définie comme la dérivée première de la fonction d'échelle, les extrema locaux de la transformée correspondent aux discontinuités d'un signal à chaque échelle. Ces extrema sont appelés maxima d'ondelettes.

La fonction d'ondelette $\psi^{1}(t)$ est définie par :

$$
\psi^{1}(t)=\frac{d \zeta(t)}{d t}
$$

$\zeta(t)$ étant la fonction d'échelle.

Notons :

$$
\psi_{e}^{1}(t)=\frac{1}{e} \psi^{1}\left(\frac{t}{e}\right)
$$

où $\psi_{e}^{1}(t)$ est la dilatation par le facteur d'échelle e de la fonction d'ondelette $\psi^{1}(t)$.

La transformée en ondelettes d'un signal $\mathrm{s}(\mathrm{t})$ à l'échelle e et au temps $\mathrm{t}$, calculée grâce à l'ondelette $\psi^{1}(t)$, est définie par :

$$
\begin{aligned}
W_{e}^{1}(t) & =s * \psi_{e}^{1}(t) \\
& =s *\left(e \frac{d \zeta_{e}}{d t}\right)(t)
\end{aligned}
$$

En utilisant la commutativité de la convolution et de la dérivation, la relation 3 peut s'écrire sous la forme :

$$
\begin{aligned}
W_{e}^{1}(t) & =e \frac{d}{d t}\left(s * \zeta_{e}\right)(t) \\
& =\left(e \frac{d s}{d t}\right) * \zeta_{e}(t)
\end{aligned}
$$

Dans notre cas, le signal à segmenter n'est pas temporel mais spatial. La relation 4 devient donc :

$$
\begin{aligned}
W_{e}^{1}(x) & =e \frac{d}{d x}\left(s * \zeta_{e}\right)(x) \\
& =\left(e \frac{d s}{d x}\right) * \zeta_{e}(x)
\end{aligned}
$$

où $\mathrm{x}$ représente l'abscisse curviligne.

En d'autres termes, la relation 5 s'interprète comme une dérivation du signal suivie d'un lissage par la fonction $\zeta_{e}(x)$ à l'échelle e. Plus l'échelle est grande, plus la convolution avec $\zeta_{e}(x)$ fait disparaître les variations rapides; les extrema sont alors dus aux variations lentes et significatives du signal.

Mallat et Zhong [SS92] ont montré que les minima locaux de $W_{e}^{1} s(x)$ ne correspondent pas à d'importantes variations du signal, mais à un point d'inflexion de la fonction $\mathrm{s}^{*} \zeta_{e}(x)$. En revanche, les maxima locaux de $\left|W_{e}^{1} s(x)\right|$ traduisent des discontinuités du signal observées à différentes échelles.

Le signal étudié étant discret, la transformation en ondelettes doit, elle aussi, être discrète.

Pour permettre un calcul numérique rapide, il est imposé à l'échelle de varier selon la séquence dyadique $\left(2^{j}\right)_{j \in Z}$. Meyer [(Y.90] et Daubechies et al [ISJ91] ont étudié les principales propriétés de 
la transformation dyadique en ondelettes et expliquent sous quelles conditions elle est redondante ou orthogonale, et stable.

Soit $\psi_{2^{j}}^{1}(x)$ la dilatation de la fonction d'ondelettes $\psi^{1}(x)$ par le facteur $2^{j}$ :

$$
\psi_{2^{j}}^{1}(x)=\frac{1}{2^{j}} \psi^{1}\left(\frac{x}{2^{j}}\right)
$$

La transformée en ondelettes $W_{2^{j}}^{1} s(x)$ du signal $\mathrm{s}(\mathrm{x})$, à l'échelle $2^{j}$ et à la distance $\mathrm{x}$, est définie par le même produit de convolution que précédemment, à la seule différence que :

$$
\begin{aligned}
W_{2^{j}}^{1} s(x) & =s * \psi_{2^{j}}^{1}(x) \\
& =s *\left(2^{j} \frac{d \zeta_{j}}{d x}\right)(x) \\
& =\left(2^{j} \frac{d s}{d x}\right) * \zeta_{j}(x)
\end{aligned}
$$

Rechercher les variations significatives de $\mathrm{s}(\mathrm{x})$ revient donc à présent à identifier les maxima locaux de $\left|W_{2^{j}}^{1} s(x)\right|$. La figure 27 (b) présente le résultat de la transformée pour une décomposition sur 11 échelles d'un signal simulé (figure 27 (a)).

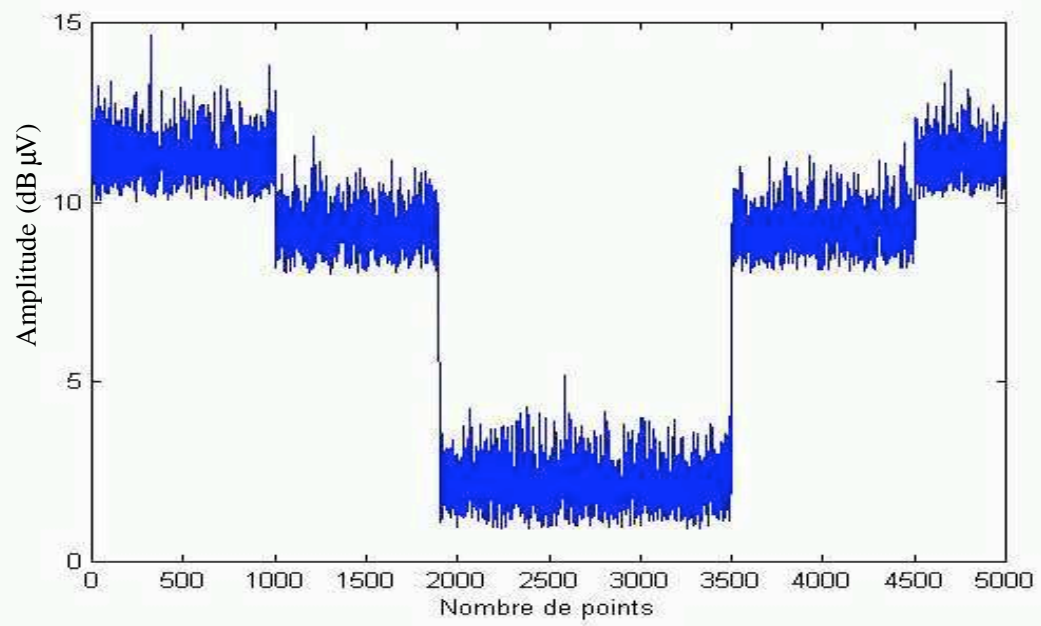

(a)

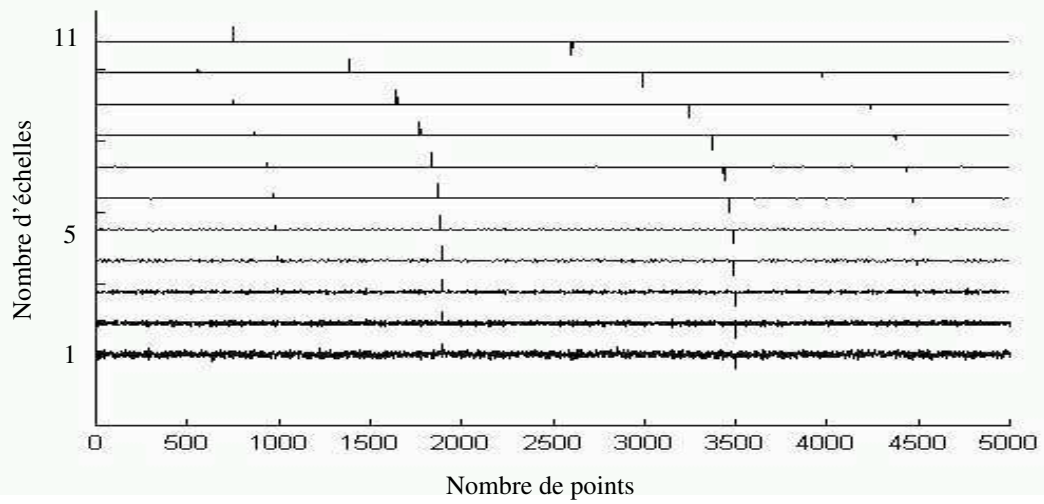

(b)

figure 9 - Segmentation d'un signal sur 11 échelles

Concrètement, cette représentation des maxima d'ondelettes contient les positions et les valeurs de $W_{e}^{1} s(x)$ pour chaque échelle quand $\left|W_{e}^{1} s(x)\right|$ atteint un maximum local. Dans notre application, il faut retenir l'échelle qui fournit les maxima permettant de segmenter le signal selon ses variations lentes et significatives. Pour le signal proposé qui présente quatre discontinuités d'amplitudes différentes, choisir 
la dixième échelle permet d'identifier les cinq intervalles du signal, alors que la onzième ne considère que les deux discontinuités principales. Pour obtenir la position de ces ruptures du signal à partir des maxima d'ondelettes, il est nécessaire de remonter fréquentiellement la position des maxima. En effet, ils subissent un décalage de position dû à la transformation qui effectue un filtrage, donc un déphasage du signal. Cette étape porte le nom de chaînage [PYRC01]. Comme cela vient d'être souligné, le choix de l'échelle permettant d'initialiser le processus de chainage est le paramètre essentiel de cette technique de segmentation. En effet, si une échelle trop fine est choisie, des ruptures non significatives du signal seront retenues et il ne sera pas possible d'associer à certaines d'entre elles des changements notables de combinaisons d'interactions électromagnétiques. A l'opposé, si une échelle trop grossière est choisie, il y a le risque de ne pas prendre en compte des modifications significatives de combinaisons d'interactions. Une étude statistique a conduit à l'élaboration d'un critère permettant d'identifier l'échelle optimale pour notre application. Il repose sur la stabilité des intervalles segmentés par rapport à la dispersion du signal total [(P.01] ce qui est traduit par le rapport $\sigma_{i} / \sigma_{s}\left(\sigma_{i}\right.$ : écart type de l'intervalle considéré et $\sigma_{s}$ : écart type du signal entier).

\subsection{Principe de vérification de l'hypothèse}

L'objet de ce paragraphe est de présenter la vérification de la validité de l'hypothèse, formulée dans le paragraphe 2, qui est la base de notre méthode d'optimisation pour la prédiction de zone de couverture. Rappelons cette hypothèse : il existe une très forte corrélation entre les variations lentes de signaux mesurés sur le terrain et les mécanismes de propagation des ondes radioélectriques. La technique utilisée pour valider cette hypothèse consiste à vérifier qu'il y a correspondance entre les variations du signal mesuré et la partition spatiale. Dans ce but, l'algorithme de segmentation (paragraphe 3.1) est appliqué pour détecter les changements de comportement des signaux mesurés.

Pour présenter cette technique, dont l'organigramme est proposé sur la figure 28 , un exemple simple est traité en détail.

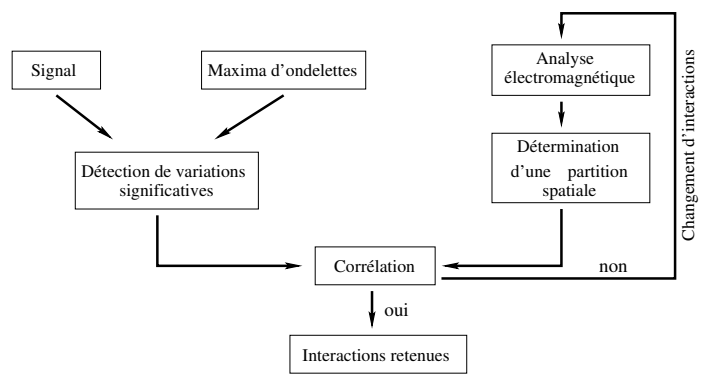

figure 10 - Organigramme de validation de l'hypothèse

Considérons le signal de la figure 30 mesuré sur le parcours matérialisé par des croix blanches sur la figure 29 (a), à une fréquence de $2 \mathrm{GHz}$.

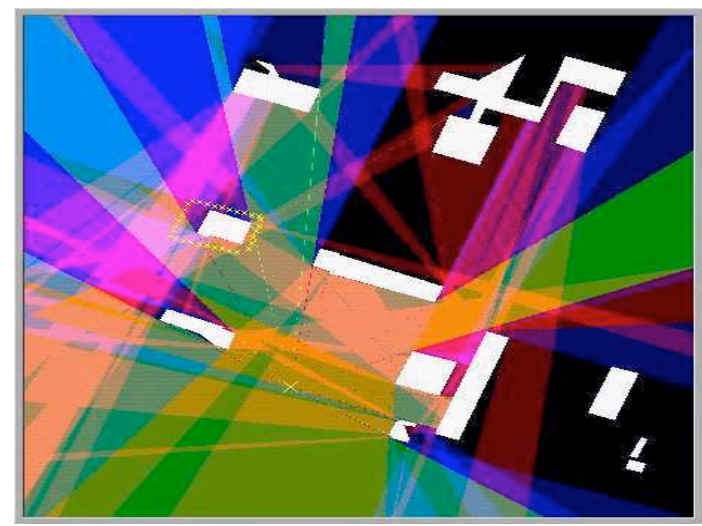

(a)

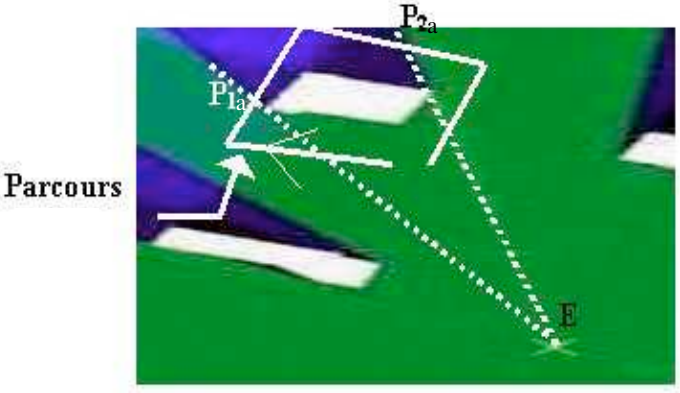

(b)

figure 11 - (a) Partition spatiale (b) Zoom sur le parcours de mesures

Après application de la segmentation sur ce signal, trois intervalles sont identifiés dont les limites 


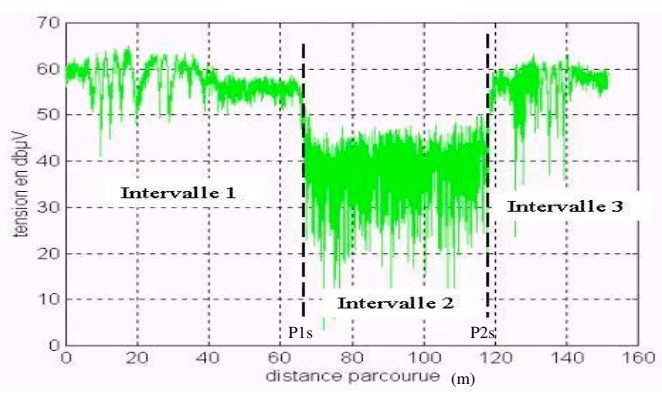

figure 12 - Signal segmenté (suburbain)

sont aux points P1s et P2s d'abscisses curvilignes $65 \mathrm{~m}$ et $117 \mathrm{~m}$ (figure 30).

Il est ensuite nécessaire d'identifier les paramètres du logiciel d'analyse électromagnétique (nombre et nature des interactions électromagnétiques) permettant d'obtenir une partition correcte par rapport à cette segmentation. La figure 29 (a) présente la partition du site étudié obtenue par l'analyse électromagnétique avec une diffraction et une réflexion.

Les résultats donnés sur le parcours étudié par cette analyse ne correspondent pas à ceux obtenus par la segmentation du signal mesuré. En effet, les éléments de la partition traversés par le parcours sont trop nombreux; la partition est donc trop fine. Il est ainsi nécessaire de diminuer le nombre d'interactions électromagnétiques à considérer.

La figure 29 (b) montre le zoom (rectangle en pointillé blanc sur la figure 29 (a)) de la partition obtenue pour une diffraction et aucune réflexion. On constate que le parcours traverse alors deux éléments de la partition, à savoir une région en visibilité de l'émetteur et la zone d'ombre du bâtiment caractérisée par le phénomène de diffraction. Les abscisses curvilignes des deux points notés P1a et P2a fournis par le logiciel d'analyse électromagnétique sont égaux à $70 \mathrm{~m}$ et $120 \mathrm{~m}$. En comparant les abscisses des points $\mathrm{P} 1 \mathrm{~s}$ et $\mathrm{P} 2 \mathrm{~s}$ issus de la segmentation et $\mathrm{P} 1 \mathrm{a}$ et $\mathrm{P} 2 \mathrm{a}$, on note une différence de l'ordre de quelques mètres. Toutefois, celle-ci est jugé acceptable compte tenu, notamment, de la précision des bases de données de terrain qui est de trois mètres [(C.81]. Ainsi, sur cet exemple simple, l'hypothèse est vérifiée : les ruptures détectées dans le signal mesuré correspondent effectivement à des changements d'interactions en choisissant comme paramètres du logiciel d'analyse électromagnétique une diffraction et aucune réflexion.

Pour un cas complexe correspondant à un signal mesuré (figure 31) en contexte microcellulaire dans Paris (France), le tableau I présente l'évolution du nombre d'intervalles constituant le parcours, obtenus par traitement de la partition spatiale en fonction de la combinaison d'interactions choisie dans l'analyse électromagnétique. La référence, est comme précédemment, le nombre de tronçons obtenus par segmentation du signal mesuré qui est égal à 203.

\begin{tabular}{|c|c|}
\hline Interactions & Nombre de segments \\
\hline 0R1D & 65 \\
\hline 0R2D & 124 \\
\hline 0R3D & 142 \\
\hline 0R4D & 149 \\
\hline 1R4D & 200 \\
\hline 2R4D & 220 \\
\hline 2R2D & 213 \\
\hline 3R3D & 243 \\
\hline
\end{tabular}

tableau I - Evolution du nombre de segments constituant le parcours en fonction des interactions

. Ce tableau met en évidence que pour la configuration microcellulaire étudiée, il est nécessaire de considérer quatre diffractions et une réflexion; la différence entre la valeur de référence et celle obtenue par analyse électromagnétique est alors de $1 \%$. En outre, dans ce cas, la différence de position des 


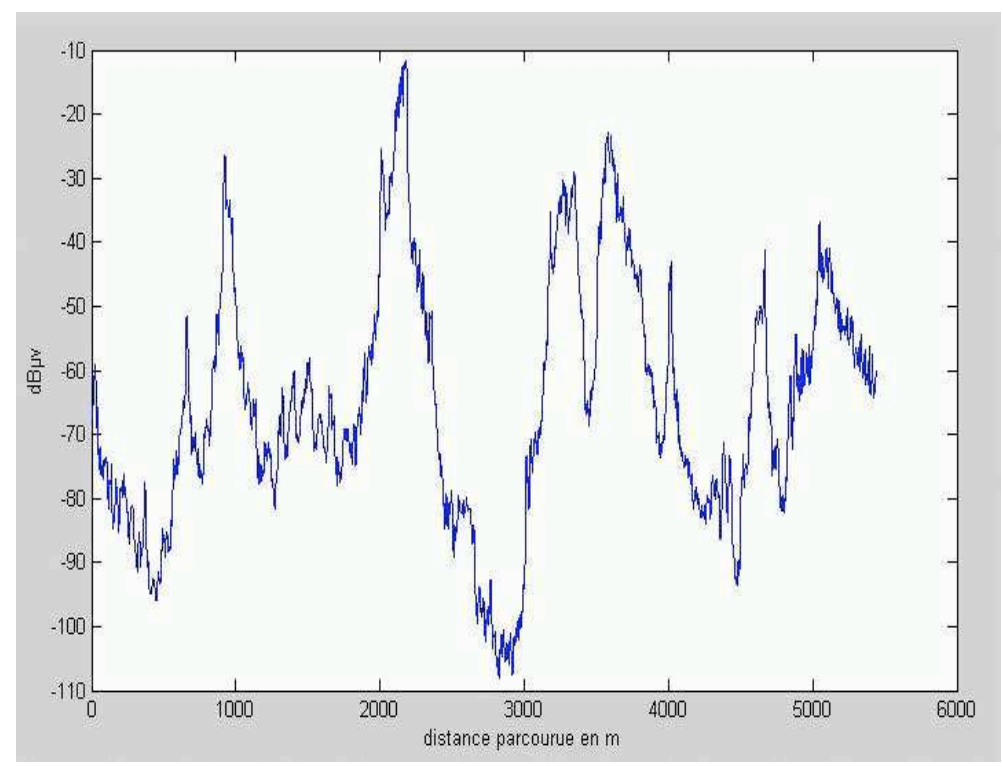

figure 13 - Signal segmenté (Paris)

extrémités des segments entre les deux démarches est, à nouveau, de quelques mètres. Comme cela sera montré dans le paragraphe 5 , cette précision est suffisante pour notre application.

De nombreux signaux dans des configurations variées ont été traités d'une façon analogue permettant ainsi de vérifier statistiquement notre hypothèse.

Remarque : sur cette base, il devient possible d'établir une méthode d'apprentissage visant à déterminer pour un type d'environnement particulier, le couple nombre de réflexions - nombre de diffractions donnant la partition spatiale optimale par rapport à de nombreux signaux mesurés. Ainsi, pour une zone géographique dont le type aurait déjà été étudié, des signaux mesurés seraient inutiles pour déterminer les paramètres de l'analyse électromagnétique. Il suffirait de considérer ceux associés à l'environnement type déjà étudié.

\section{Calcul effectif de la zone de couverture}

\subsection{Principe}

Après cette vérification de notre hypothèse, nous pouvons affirmer que notre logiciel d'analyse électromagnétique engendre des partitions spatiales constituées d'éléments dans lesquels les variations du niveau moyen du signal reçu sont faibles.

Notre optimisation du temps de calcul d'une zone de couverture consiste à appliquer un modèle de propagation sur seulement quelques points par élément de la partition spatiale, et à extrapoler le niveau reçu à tout l'élément. Le processus retenu suit donc l'organigramme de la figure 32 . Son principe est le suivant : à partir d'un environnement type et de la combinaison optimale d'interactions (nombre de réflexions - nombre de diffractions) correspondante, nous obtenons, via le logiciel d'analyse électromagnétique, la partition spatiale optimale. Nous appliquons alors un modèle de propagation sur seulement quelques points de chaque élément de partition, puis le niveau moyen estimé est extrapolé à tout l'élément. Pour mettre en oeuvre cet organigramme, il reste ainsi à déterminer le nombre de points d'application du modèle de propagation et leurs positions dans chaque élément de la partition.

\subsection{Nombre de points par élément de partition}

Ce nombre de points est un des paramètres clés des performances de notre méthode. En effet, il doit être minimum pour réduire le temps de calcul par rapport à une méthode classique de prédiction, tout en étant suffisamment grand pour assurer une bonne estimation de la puissance reçue dans l'élément considéré.

Pour l'identifier, notre principe consiste à rechercher le nombre de points nécessaires à l'estimation de la puissance médiane d'un segment obtenu par analyse multiéchelle (paragraphe 3.1), avec une incertitude acceptable. Elle a été fixée arbitrairement à $3 \mathrm{~dB}$ car, comme cela sera montré dans le paragraphe 


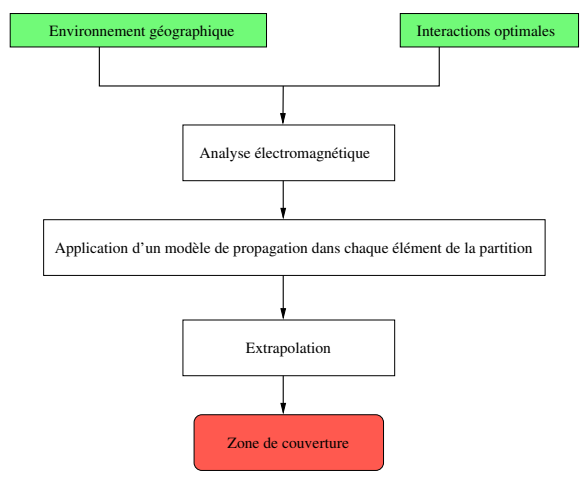

figure 14 - Organigramme de la phase d'application

5 , cette valeur conduit à des résultats satisfaisants. L'évolution de l'erreur d'estimation de la puissance médiane en fonction du nombre de points considérés pour le calcul est présentée sur la figure 33.

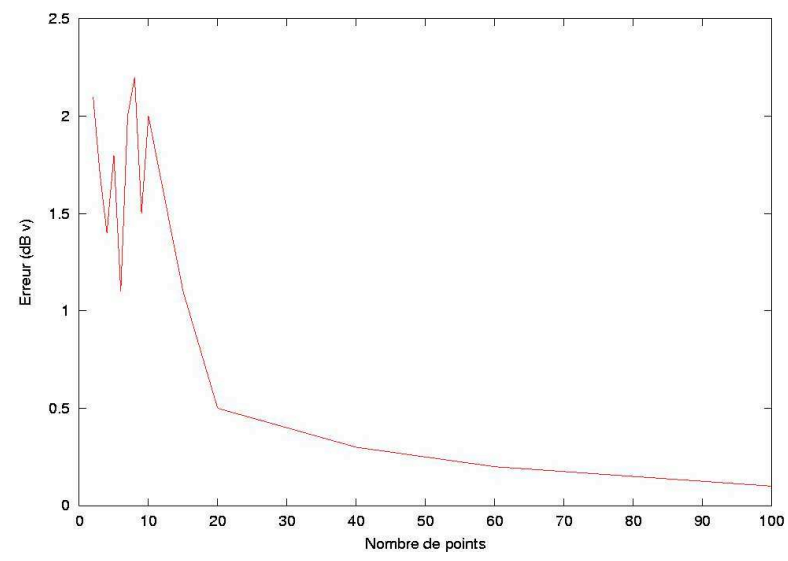

figure 15 - Evolution de l'erreur d'estimation en fonction du nombre de points considérés par segment

Cette courbe résulte d'une étude statistique fiable portant sur de nombreux signaux représentant plusieurs dizaines de milliers de points de mesures. Il apparaît très clairement que deux points suffisent pour estimer le niveau d'un tronçon du signal avec une erreur largement inférieure à $3 \mathrm{~dB}$. On pouvait s'attendre à ce type de résultat puisqu'une des propriétés principales du traitement par maxima d'ondelettes est d'assurer la stabilité du signal sur chacun de ses tronçons. En outre, au cours de cette étude, il a été montré que ces deux points doivent être disposer uniformément par intervalle afin de prendre en compte d'éventuelles variations non significatives du signal.

En extrapolant le raisonnement en deux dimensions, nous considérons deux points d'application répartis uniformément dans chaque élément de la partition spatiale afin d'y estimer la puissance.

\subsection{Modèle de propagation et extrapolation}

Conformément à l'organigramme de la figure 32, après avoir appliqué l'analyse électromagnétique sur la zone géographique étudiée, nous disposons d'une partition dont chaque région est caractérisée par une combinaison d'interactions. La figure 34 (a) illustre une telle partition pour le quartier de Paris dans lequel le signal de la figure 31 a été mesuré.

Pour prédire la puissance reçue dans cette zone géographique, un modèle de propagation quelconque est alors appliqué pour chaque couple de points par élément de la partition, identifiés précédemment. Pour chaque élément, la moyenne de ces deux estimations est alors affectée aux points du maillage régulier, inclus dans cet élement, qui serait utilisé dans une méthode classique de prédiction de couverture. Nous obtenons ainsi une estimation de la couverture comparable à celle fournie par une technique classique (figure 34 (b)) ; sur cette figure, l'évolution de la puissance reçue est indiquée par des niveaux de gris.

Toutefois, nous avons remarqué que les éléments de partition en visibilité directe de l'émetteur sont, en général, assez grands, comme l'illustre la figure 34 (a); une extrapolation uniforme du niveau moyen 


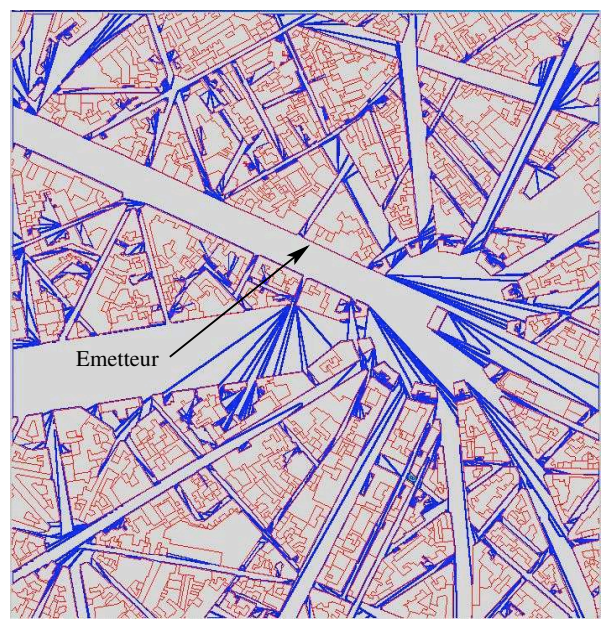

(a)

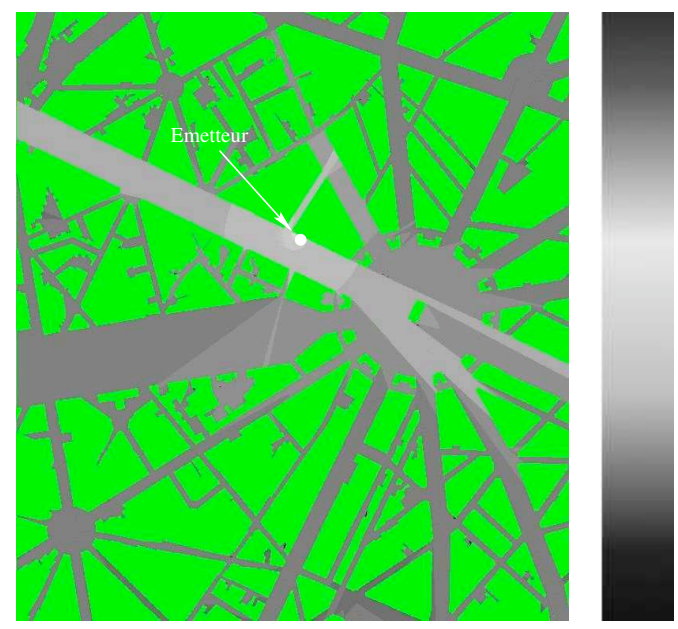

(b)

figure 16 - (a) Partition spatiale (b) Zone de couverture

estimé y implique alors une erreur de prédiction assez importante. Pour éliminer cet inconvénient, nous appliquons le modèle en espace libre dans ces régions, sur chaque point de la grille régulière.

\section{Résultats}

Ce paragraphe expose l'évaluation des performances de notre méthode d'optimisation en termes de précision et de temps de calcul. Nous mettons aussi en évidence la possibilité de privilégier un compromis entre une couverture de qualité et un gain important en temps de calcul. Les résultats présentés sont relatifs à deux configurations (microcellulaire et petite cellule) et ont été obtenus avec un modèle scalaire dit $\mu \mathrm{G}$ [(X.00]. Pour les deux configurations, les trois versions de notre méthode (2DH, 2DV et 2,5D) sont évaluées. Notons que notre méthode fonctionne pour des fréquences supérieures au giga hertz, contrainte liée à la prise en compte du phénomène de diffraction. En effet les zones de transitions autour des frontières optiques doivent être très étroites afin de ne considérer le phénomène de diffraction que dans les zones d'ombre (cf figure 4). Tous les résultats de temps de calcul ont été obtenus avec un processeur $1,6 \mathrm{GHz}$.

\subsection{Configuration microcellulaire}

Cette configuration correspond à un émetteur situé à $7 \mathrm{~m}$ au dessus du sol, c'est à dire nettement inférieure à la hauteur moyenne des toits dans ce quartier de l'arc de triomphe à Paris. La fréquence considérée est voisine de 1,8 GHz. Le tableau II présente les résultats associés à cette configuration.

\begin{tabular}{|c|c|c|c|c|c|c|c|c|}
\hline \multirow[b]{2}{*}{ Versions } & \multirow[b]{2}{*}{ Interactions } & \multirow[b]{2}{*}{$\phi(50 \%)$} & \multirow[b]{2}{*}{$\phi(90 \%)$} & \multirow[b]{2}{*}{ Couverture } & \multicolumn{3}{|c|}{ Temps de calculs } & \multirow{2}{*}{ Réduction du nombre de points } \\
\hline & & & & & Traitements spécifiques & Modèle & temps total & \\
\hline \multirow{6}{*}{ 2DH } & OR1D & $1,15 \mathrm{~dB}$ & $3,5 \mathrm{~dB}$ & $25,13 \%$ & $9 \mathrm{~s} \rightarrow 1$ & $\mathrm{t} 1$ & $9 \mathrm{~s}+\mathrm{t} 1$ & $98,72 \%$ \\
\hline & OR2D & $1,43 \mathrm{~dB}$ & $5,88 \mathrm{~dB}$ & $56,38 \%$ & $12 \mathrm{~s} \rightarrow>1,33$ & $\mathrm{t} 2$ & $12 \mathrm{~s}+\mathrm{t} 2$ & $96,79 \%$ \\
\hline & OR3D & $1,65 \mathrm{~dB}$ & $5,93 \mathrm{~dB}$ & $72,00 \%$ & $20 \mathrm{~s} \rightarrow 2,22$ & $\mathrm{t} 3$ & $20 \mathrm{~s}+\mathrm{t} 3$ & $92,30 \%$ \\
\hline & OR4D & $1,46 \mathrm{~dB}$ & $5,53 \mathrm{~dB}$ & $79,39 \%$ & $33 \mathrm{~s}->3,66$ & $\mathrm{t} 4$ & $33 \mathrm{~s}+\mathrm{t} 4$ & $88,27 \%$ \\
\hline & 1R4D & $0,96 \mathrm{~dB}$ & $2,19 \mathrm{~dB}$ & $81,71 \%$ & $2 \mathrm{mn} 4 \mathrm{~s} \rightarrow 13,77$ & $\mathrm{t} 5$ & $2 \mathrm{mn} 4 \mathrm{~s}+\mathrm{t} 5$ & $83,28 \%$ \\
\hline & 2R4D & $0,96 \mathrm{~dB}$ & $2,17 \mathrm{~dB}$ & $82,06 \%$ & $6 \mathrm{mn} 8 \mathrm{~s} \rightarrow>40,88$ & t6 & $6 \mathrm{mn} 8 \mathrm{~s}+\mathrm{t} 6$ & $81,29 \%$ \\
\hline $2 \mathrm{DV}$ & 0R4D & $0,92 \mathrm{~dB}$ & $3,3 \mathrm{~dB}$ & $60,75 \%$ & $37 \mathrm{~s} \rightarrow 4,11$ & $\mathrm{t} 7$ & $37 s+t 7$ & $88,48 \%$ \\
\hline $2.5 \mathrm{D}$ & OR4D & $1,08 \mathrm{~dB}$ & $4,55 \mathrm{~dB}$ & $84,24 \%$ & $54 \mathrm{~s} \rightarrow>6$ & t8 & $54 \mathrm{~s}+\mathrm{t} 8$ & $86,38 \%$ \\
\hline
\end{tabular}

tableau II - Performances pour une configuration microcellulaire

Dans un premier temps, nous évaluons la qualité de la couverture obtenue en fonction des combinaisons d'interactions considérées dans l'analyse électromagnétique, grâce à trois critères. Les deux premiers sont les valeurs à $50 \%$ et $90 \%$ de la fonction de répartition $\phi(\mathrm{x})$ des erreurs d'estimation. Celles-ci résultent de la différence des puissances calculées selon notre méthode et selon la démarche 
classique, pour chaque point du maillage régulier considéré. Ici, le pas du maillage est conforme à celui utilisé par les opérateurs en milieu urbain, à savoir cinq mètres. Le troisième critère est le taux de couverture de notre méthode, c'est à dire le pourcentage de points pour lesquels notre méthode estime une puissance. En effet, plus la combinaison d'interactions considérée dans l'analyse électromagnétique est importante, plus les ondes simulées pourront se propager dans des endroits difficilement atteignables.

Dans un deuxième temps, nous évaluons la rapidité de notre méthode : dans ce but, et pour chaque combinaison d'interactions, nous présentons les temps de calcul de notre méthode en distinguant le temps mis par nos traitements spécifiques (analyse électromagnétique et extrapolation) et celui du modèle de propagation. Toutefois, comme notre méthode est indépendante du modèle et que le type (scalaire, vectoriel, ...) de ce dernier est essentiel pour le temps de prédiction de couverture, nous indiquons, la réduction du nombre de points d'application du modèle, obtenue par notre technique par rapport à la méthode classique. En effet, pour un facteur de réduction constant, notre méthode est d'autant plus efficace en terme de gain en temps de calcul que le modèle est complexe. Notons que la colonne traitements spécifiques comporte deux informations, le temps de calcul lui même ainsi que le coefficient multiplicatif par rapport au temps de référence qui est celui de la configuration 0R1D (9 secondes). Par exemple, la combinaison 0R4D prends 4,11 fois plus de temps soit 37 secondes. Pour évaluer le gain en temps de calcul de notre méthode, le temps de référence est celui mis par une technique classique qui, pour les 30601 récepteurs fictifs du maillage régulier considéré, est égal à 11 minutes pour le modèle scalaire $\mu \mathrm{G}$, et à environ une heure jusqu'à plusieurs jours, pour un modèle 3D vectoriel selon la combinaison d'interactions.

Tout d'abord, pour la version 2DH, l'analyse du tableau II met en évidence la très nette augmentation du taux de couverture lors de l'accroissement du nombre de diffraction alors que la précision n'évolue pas, excepté dans le cas très simple d'une diffraction. Cela signifie que notre méthode est précise dans tous les cas, mais, pour être opérationnelle, elle doit être appliquée avec quatre diffractions. Concernant la prise en compte de réflexions, on observe que cela n'augmente pas le taux de couverture mais améliore sensiblement la précision. Concrètement, cela indique qu' une fois l'onde arrivée dans une rue grâce à une diffraction, considérer une réflexion permet d'affiner la couverture de cette rue en créant de petites zones de réflexions.

Si l'on privilégie la précision, il apparaît que la combinaison optimale d'interactions est une réflexion associée à quatre diffractions : d'une part, on obtient une précision très satisfaisante car $90 \%$ des erreurs d'estimation de la puissance reçue sont inférieures à $2,2 \mathrm{~dB}$; d'autre part, pour un taux de couverture proche de $82 \%$, la réduction du nombre de points d'application du modèle est voisine de $85 \%$.

En cherchant à optimiser le compromis entre la précision et le temps de calcul, on retiendra la combinaison 0R4D qui permet de diviser les temps de traitements par 4 au détriment d'une augmentation de $3 \mathrm{~dB}$ de l'erreur à $90 \%$.

Dans les deux cas, il faut noter que le taux de couverture est assez éloigné de $100 \%$. Cela s'explique par la présence de nombreuses cours intérieures (figure 35) qui ne sont pas atteintes dans la version horizontale, et qui demanderaient un nombre très important de diffractions pour l'être dans la version verticale.

Compte tenu de la rapidité des traitements propres à notre méthode, l'optimisation en temps de calcul est principalement liée à la réduction du nombre de points d'application du modèle utilisé. Ainsi, pour les modèles scalaire et vectoriel considérés, le gain en temps est respectivement voisin d'un facteur 4 et 80 par rapport à une technique classique.

Par ailleurs, il est important de noter que cette dernière combinaison d'interactions (0R4D) pour une analyse électromagnétique en vertical donne un taux de couverture très médiocre. Ce résultat indique que dans une configuration microcellulaire, la propagation des ondes s'effectue essentiellement dans un plan horizontal conformément à la littérature. L'analyse électromagnétique en 2,5D n'est ainsi pas plus intéressante que la version horizontale, mise à part si l'on veut étudier le phénomène de brouillage intercellules. Dans ce cas, il suffit de lancer deux calculs de couverture pour deux emplacements différents de l'émetteur et de superposer les deux résultats afin d'observer ce phénomène et ainsi l'influence des trajets verticaux provenant des cellules voisines.

\subsection{Configuration petite-cellule}

Contrairement à la configuration précédente, l'émetteur est situé sur un toit ; la fréquence est toujours voisine de 1,8 GHz. En suivant un raisonnement analogue au précédent sur la propagation des ondes, tout laisse à penser qu'une analyse électromagnétique verticale est la plus appropriée. En effet, dans ce type de configuration l'essentiel des ondes se propage dans des plans verticaux passant par l'émetteur 


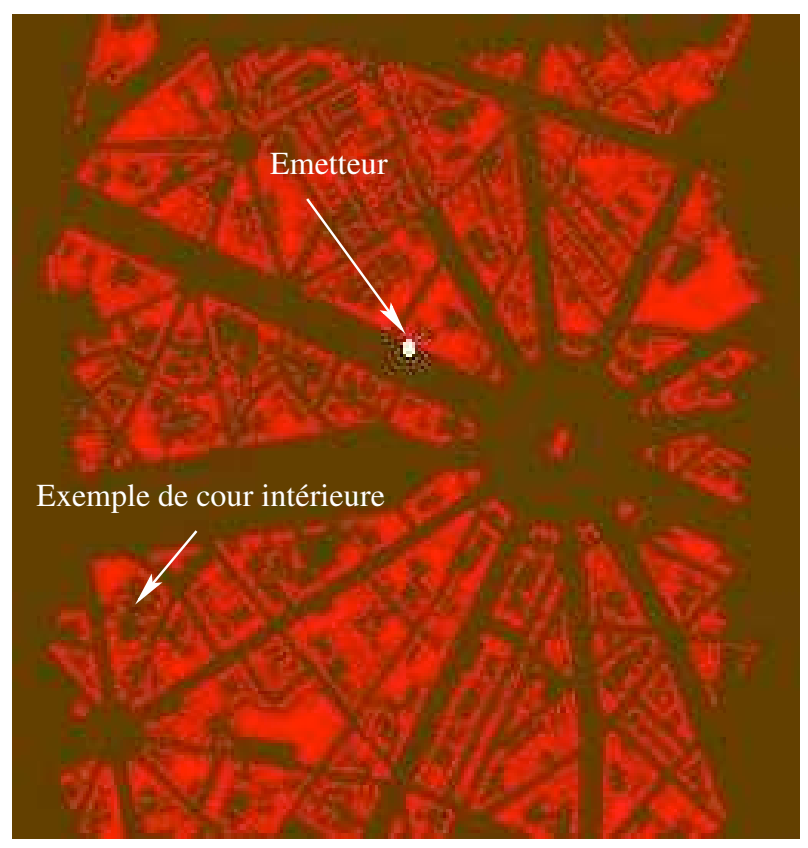

figure 17 - Vue de dessus de la scène (bâtiments en gris et sol en noir)

[(J.88, (C.00]. L'analyse du tableau III le confirme.

\begin{tabular}{|c|c|c|c|c|c|c|c|c|}
\hline & \multirow{2}{*}{\multicolumn{3}{|c|}{ Temps de calculs }} & \multirow{3}{*}{ Réduction du nombre de points } \\
\hline & & & & & & & & \\
\hline Versions & Interactions & $\phi(50 \%)$ & $\phi(\mathbf{9 0 \%})$ & Couverture & Traitements spécifiques & Modèle & temps total & \\
\hline \multirow{8}{*}{ 2DV } & OR1D & $1,24 \mathrm{~dB}$ & $4,81 \mathrm{~dB}$ & $29,23 \%$ & $1 \mathrm{mn} 18 \mathrm{~s} \rightarrow>1$ & $\mathrm{t} 1$ & $1 \mathrm{mn} 18 \mathrm{~s}+\mathrm{t} 1$ & $97,04 \%$ \\
\hline & OR2D & $1,15 \mathrm{~dB}$ & $5,01 \mathrm{~dB}$ & $54,50 \%$ & $1 \mathrm{mn} 49 \mathrm{~s} \rightarrow 1,40$ & $\mathrm{t} 2$ & $1 \mathrm{mn} 49 \mathrm{~s}+\mathrm{t} 2$ & $92,61 \%$ \\
\hline & OR3D & $1,1 \mathrm{~dB}$ & $4,91 \mathrm{~dB}$ & $73,41 \%$ & $2 \mathrm{mn} 31 \mathrm{~s} \rightarrow 1,94$ & $\mathrm{t} 3$ & $2 \mathrm{mn} 31 \mathrm{~s}+\mathrm{t} 3$ & $89,32 \%$ \\
\hline & OR4D & $1,08 \mathrm{~dB}$ & $4,80 \mathrm{~dB}$ & $84,11 \%$ & $3 \mathrm{mn} 49 \mathrm{~s}->2,93$ & $\mathrm{t} 4$ & $3 \mathrm{mn} 49 \mathrm{~s}+\mathrm{t} 4$ & $87,10 \%$ \\
\hline & 1R1D & $1,17 \mathrm{~dB}$ & $4,68 \mathrm{~dB}$ & $36,12 \%$ & $1 \mathrm{mn} 50 \mathrm{~s} \rightarrow>1,41$ & $\mathrm{t} 5$ & $1 \mathrm{mn} 50 \mathrm{~s}+\mathrm{t} 5$ & $95,35 \%$ \\
\hline & 1R2D & $1,12 \mathrm{~dB}$ & $4,83 \mathrm{~dB}$ & $58,92 \%$ & $2 \mathrm{mn} 42 \mathrm{~s}->2,08$ & t6 & $2 \mathrm{mn} 42 \mathrm{~s}+\mathrm{t} 6$ & $92,00 \%$ \\
\hline & 1R3D & $1,12 \mathrm{~dB}$ & $4,84 \mathrm{~dB}$ & $76,20 \%$ & $4 \mathrm{mn} 18 \mathrm{~s} \rightarrow 3,62$ & $\mathrm{t} 7$ & $4 \mathrm{mn} 18 \mathrm{~s}+\mathrm{t} 7$ & $88,97 \%$ \\
\hline & 1R4D & $1,12 \mathrm{~dB}$ & $4,82 \mathrm{~dB}$ & $88,30 \%$ & $7 \mathrm{mn} 55 \mathrm{~s} \rightarrow 6,09$ & t8 & $7 \mathrm{mn} 55 \mathrm{~s}+\mathrm{t} 8$ & $90,30 \%$ \\
\hline $2 \mathrm{DH}$ & OR4D & $2,35 \mathrm{~dB}$ & $9,17 \mathrm{~dB}$ & $32,48 \%$ & $1 \mathrm{mn} 5 \mathrm{~s} \rightarrow 0,83$ & t9 & $1 \mathrm{mn} 5 \mathrm{~s}+\mathrm{t} 9$ & $95,76 \%$ \\
\hline $2.5 \mathrm{D}$ & OR4D & $1,15 \mathrm{~dB}$ & $5,00 \mathrm{~dB}$ & $85,20 \%$ & $5 \mathrm{mn} 12 \mathrm{~s} \rightarrow 4$ & $\mathrm{t} 10$ & $5 \mathrm{mn} 12 \mathrm{~s}+\mathrm{t} 10$ & $88,32 \%$ \\
\hline
\end{tabular}

tableau III - Performances pour une configuration petite cellule

Notons que le temps de référence mis par une technique classique utilisant le modèle $\mu \mathrm{G}$ présenté précédemment est de 15 minutes, pour une grille de 84000 récepteurs fictifs.

On peut remarquer que seule une étude dans des plans verticaux permet d'obtenir une zone de couverture de taille significative pour un nombre acceptable d'interactions. Concernant l'influence des combinaisons d'interactions sur la précision de l'estimation, les mêmes conclusions qu'en microcellulaire peuvent être tirées : l'augmentation du nombre de diffractions permet de couvrir une surface de plus en plus importante, alors que celle du nombre de réflexions a pour conséquence de réduire l'erreur d'estimation, et ceci pour les raisons évoquées précédemment.

Globalement, comme pour la configuration précédente, la meilleure simulation est obtenue en considérant quatre diffractions, avec une couverture et une réduction du nombre de points d'application égales respectivement à $85 \%$ et $87 \%$, et une bonne précision puisque $90 \%$ des erreurs sont inférieures à 4,8 $\mathrm{dB}$. Toutefois, il faut noter que le temps lié aux traitements spécifiques de notre méthode est plus long qu'en microcellulaire. Cela s'explique par le fait que la zone étudiée est nettement plus dense en terme d'urbanisation et contient donc un nombre d'arêtes et de faces bien supérieure en comparaison avec la scène précédente. Le temps de calcul des traitements spécifiques étant étroitement lié à ce nombre de faces et d'arêtes, il est normal qu'il augmente.

La figure 36 permet de visualiser le résultat de la zone de couverture calculée avec ces paramètres pour l'analyse électromagnétique. 

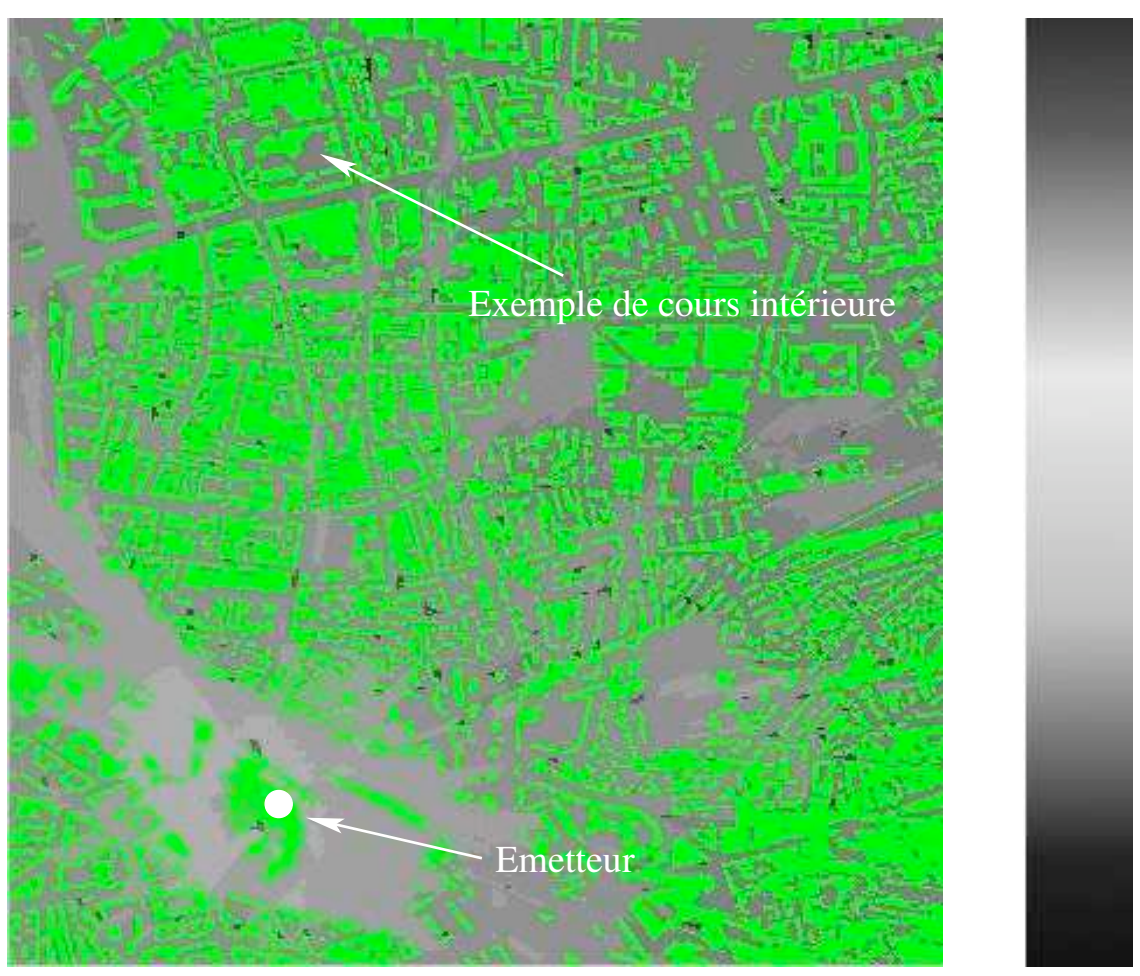

figure 18 - Visualisation de la couverture

\section{Conclusion}

Les travaux présentés dans cet article visent la réduction du temps de calcul nécessaires à la prédiction de la zone de couverture d'un émetteur. Cette méthode, indépendante du modèle de propagation, repose sur la réduction du nombre de points d'application du modèle choisi par rapport à une technique classique basée sur un maillage régulier. La méthode développée s'appuie sur l'hypothèse suivante : les variations des signaux mesurés sont directement liées aux phénomènes physiques subis par l'onde au cours de sa propagation. Ainsi, à chaque variation significative des signaux, on observe un changement de combinaison d'interactions.

La technique proposée repose sur un logiciel d'analyse électromagnétique développé au laboratoire qui permet de partitionner la zone géographique en éléments, caractérisés par une même combinaison d'interactions. Il existe trois versions considérant la propagation des ondes en 2D (horizontal ou vertical) ou en $2,5 \mathrm{D}$.

Tout d'abord, l'hypothèse précédente a été vérifiée statistiquement en montrant la forte corrélation entre la partition spatiale fournie par l'analyse électromagnétique correctement paramétrée et la segmentation par traitement multi échelle de signaux mesurés dans différentes configurations. Ensuite, le calcul effectif d'une zone de couverture a été décrit en insistant sur la détermination du nombre minimum de points à considérer, pour estimer la puissance moyenne reçue dans chaque élément de la partition de l'environnement étudié. En effet, ce nombre intervient directement sur les performances de notre méthode; il a été montré qu'il est égal à 2.

Globalement, le principe de la prédiction optimisée de la couverture, qui s'affranchit de signaux mesurés, consiste à appliquer le logiciel de partitionnement, puis à lancer le modèle de propagation sur deux points dans chaque élément de la partition obtenue. Cette partition est bien sûr déterminée avec les paramètres d'entrée optimaux (nombre de réflexion et de diffraction) qui sont fonction du type de configuration étudiée. La puissance moyenne calculée avec les deux estimations fournies par un modèle de propagation est alors affectée à tous les points d'un maillage régulier inclus dans l'élément considéré. Toutefois, il faut noter que les éléments en visiblité directe de l'émetteur sont traités différemment : un modèle de propagation en espace libre est directement appliqué sur l'ensemble des points du maillage régulier de ces régions afin de limiter les erreurs introduites par leurs grandes dimensions.

Enfin, la dernière partie de cet article présente les performances de notre méthode en termes de 
précision et de gain en temps de calcul dans deux configurations : petite cellule et micro cellule. Cette évaluation a été réalisée en comparant la méthode classique considérant une grille régulière et notre approche.

Pour une configuration micro cellulaire, il a été montré qu'une analyse électromagnétique réalisée dans un plan horizontal s'impose très nettement. Le gain en temps de calcul atteint alors un facteur trois pour un modèle scalaire, tout en assurant une excellente précision puisque $90 \%$ des erreurs d'estimation du niveau reçu sont inférieures à $2,2 \mathrm{~dB}$. Ce résultat satisfaisant s'explique par la réduction très importante du nombre de points d'application du modèle qui atteint $80 \%$. Cette valeur indique qu'en employant un modèle complexe (e.g. un modèle 3D vectoriel), le gain en temps de calcul est considérable; il atteint en effet un facteur 80.

En contexte petite cellule, où l'analyse électromagnétique verticale s'est montrée la plus performante, la précision est semblable au cas précédent et le pourcentage de réduction du nombre de points d'application du modèle est proche de $90 \%$.

En conclusion, cette méthode permet, en utilisant un modèle de propagation quelconque, d'estimer la zone de couverture d'un émetteur dans des configurations variées. Le principe de notre méthode laisse le choix à l'utilisateur de privilégier soit le gain en temps de calcul, soit la précision, soit encore un compromis entre ces deux aspects.

En outre, bien que cela n'ait pas été présenté dans cet article, notre méthode est compatible avec l'utilisation d'antennes sectorielles. Cet aspect est important car il permet de prendre en compte le phénomène de respiration des cellules, un problème nouveau mais essentiel pour la planification des systèmes dits $3 \mathrm{G}$ tels que l'UMTS. Ainsi, en fonction du traffic dans la cellule, il devient possible d'orienter la puissance émise dans une direction donnée afin d'adapter la zone de couverture de l'émetteur aux besoin du réseau.

\section{Remerciements :}

Ce travail a bénéficié du soutien technique et financier de France Telecom Recherche et Développement dans le cadre du contrat $n^{\circ} 011 \mathrm{~B} 323$.

\section{Références}

[(C.81] Boucher (C.). Les représentations planes conformes de lambert, projections en usage pour la france métropolitaine. Technical report, IGN/SGN, 1979-1980-1981.

[(C.90] Lee (C.). Mobile cellular communications systems. Mc Gray-Hill internationnal editions, New York, 1990.

[(C.00] Gonçaves (C.). A propagation model for urban microcellular systems at the UHF band. IEEE Transactions on vehicular technology, 49(4) :1294-1302, 2000.

[FYLR01] Escarieu (F.), Pousset (Y.), Aveneau (L.), and Vauzelle (R.). Outdoor and indoor channel characterization by a 3d simulation software. PIRMC, San Diego, USA, 2001.

[GA00] Athanasiadou (G.) and Nix (A.). Investigation into the sensitivity of the power predictions of a microcellular ray tracing propagation. IEEE Transactions on Vehicular Technology, 49(4) :1140-1151, 2000.

[ISJ91] Daubechies (I.), Jaffard (S.), and Journe (J.L.). A simple wilson orthogonal basis with exponential decay. SIAM J. Math. Anal, 22 :554-573, erratum 878-1991, 1991.

[(J.62] Keller (J.B.). Geometrical theory of diffraction. Journal of the Optical Society of America, 52(2) :116-130, 1962.

[(J.88] Walfisch (J.). A theorical model of UHF propagation in urban environments. IEEE Trans., 36(12) :1788-1796, 1988.

[(J.91] Deygout (J.). Correction factor for multiple knife-edge diffraction. IEEE Transactions on Antennas and Propagation, 39(8), 1991.

[(J.92] Parsons (J.). The mobile radio propagation channel. Pentech Press, 1992.

[(L.83] Boithias (L.). Propagation des ondes électromagnétiques dans l'environnement terrestre. Collection technique et scientifique des télécommunications. DUNOD, 1983.

[LYRM00] Aveneau (L.), Pousset (Y.), Vauzelle (R.), and Mériaux (M.). Physical and computer optimisation for the 3d utd model. AP2000, 2000. 
[(M.80] Hata (M.). Empirical formula for propagation loss in land mobile radio services. IEEE Transactions on Vehicular Technology, 29(3):317-325, 1980.

[(P.93] Flandrin (P.). Temps-Fréquence. Hermès, 1993.

[(P.01] Combeau (P.). Contribution à l'optimisation de la prédiction de la zone de couverture d'un émetteur dans le domaine des radiocommunications mobiles. Technical report, IRCOMSIC, 2001.

[PYRC01] Carré (P.), Pousset (Y.), Vauzelle (R.), and Fernandez (C.). Segmentation de signaux par maxima d'ondelettes : application à la prédiction de zones de couverture radioélectrique. Traitement du signal, 18(3) :195-211, 2001.

[RP74] Kouyoumjian (R.G.) and Pathak (P.H.). A uniform geometrical theory of diffraction for an edge in a perfectly conducting surface. Proc. IEEE, 62 :1448-1461, 1974.

[(S.88] Mallat (S.). Multiresolution representations and wavelets. PhD thesis, Univ. of Pennsylvania, 1988.

[SS92] Mallat (S.) and Zhong (S.). Characterization of signals from multiscale edges. IEEE Trans. of PAMI, 14(7) :710-732, 1992.

[(W.93] Lee (W.). Mobile Communications Design Fundamentals. Wiley Series in telecommunications, New York, 1993.

[(X.00] Lagrange (X.). Les réseaux radiomobiles. Hermes, 2000.

[(Y.68] Okumura (Y.). Field strength and its variability in vhf and uhf land-mobile radio service. Review of the electrical communication laboratory, 16(9-10):825-873, 1968.

[(Y.90] Meyer (Y.). Ondelettes et Opérateurs I. Hermann, 1990.

[YRL03] Pousset (Y.), Vauzelle (R.), and Aveneau (L.). Physical optimisation of ray tracing methods for coverage prediction. PSIP, Grenoble, 2003. 


\section{LEGENDES DES FIGURES}

figure 1 - Propagation par trajets multiples (E : émetteur, R : récepteur).

Multipath propagation ( $\mathrm{E}$ : transmitter, $\mathrm{R}$ : receiver).

figure 2 - $\quad$ (a) Partition spatiale (b) Signal schématique reçu sur le parcours de mesure.

(a) Spatial partition (b) Schematic signal received on the measurement route.

figure 3 - Zones réfléchies (a) et diffractées (b) par un bâtiment.

Reflected (a) and diffracted (b) areas by a building.

figure 4 - $\quad$ Limite de l'influence des ondes diffractées liée à $\alpha$.

Influence limit of the diffracted waves connected to $\alpha$.

figure 5 - $\quad$ Les différentes zones engendrées par un bâtiment pour une diffraction et une réflexion.

The different areas provided by a building for one diffraction and one reflection.

figure 6 - Résultat du regroupement.

Regrouping result.

figure 7 - (a) Balayage régulier de l'environnement par des plans verticaux (b) Analyse électromagnétique du plan noté (2) pour une diffraction et zéro réflexion.

(a) Regular scanning of the environment by vertical plans (b) Electromagnetic analysis of the plan called (2) for one diffraction and zero reflection.

figure 8 - Construction d'une partition issue de l'analyse électromagnétique en verticale.

Build of a partition due to the vertical electromagnetic analysis.

figure 9 - Segmentation d'un signal sur onze échelles.

Segmentation of a signal with eleven scales.

figure 10 - Organigramme de validation de l'hypothèse.

Organization flowchart of the hypothesis validation.

figure 11 - (a) Partition spatiale (b) Zoom sur le parcours de mesures.

(a) Spatial partition (b) Zoom on the mobile route.

figure 12 - Signal segmenté (suburbain).

Segmented signal (suburban).

figure 13 - Signal segmenté (Paris).

Segmented signal (Paris).

figure 14 - Organigramme de la phase d'application.

Organization flowchart of the application phase.

figure 15 - Evolution de l'erreur d'estimation en fonction du nombre de points considérés par segment. Evolution of the estimation error according to the number of points considered per segment.

figure 16 - (a) Partition spatiale (b) Zone de couverture.

(a) Spatial partition (b) Coverage zone.

figure 17 - Vue de dessus de la scène (bâtiments en gris et sol en noir).

Top view of the scene (buildings in grey and ground in black).

figure 18 - Visualisation de la couverture.

Vizualisation of the coverage. 


\section{LEGENDES DES TABLEAUX}

tableau I - Evolution du nombre de segments constituant le parcours en fonction des interactions. Evolution of the route segment number with respect to the interactions.

tableau II - Performances pour une configuration microcellulaire. Performances for a microcellular configuration.

tableau III - Performances pour une configuration petite cellule.

Performances for a little cellular configuration.. 


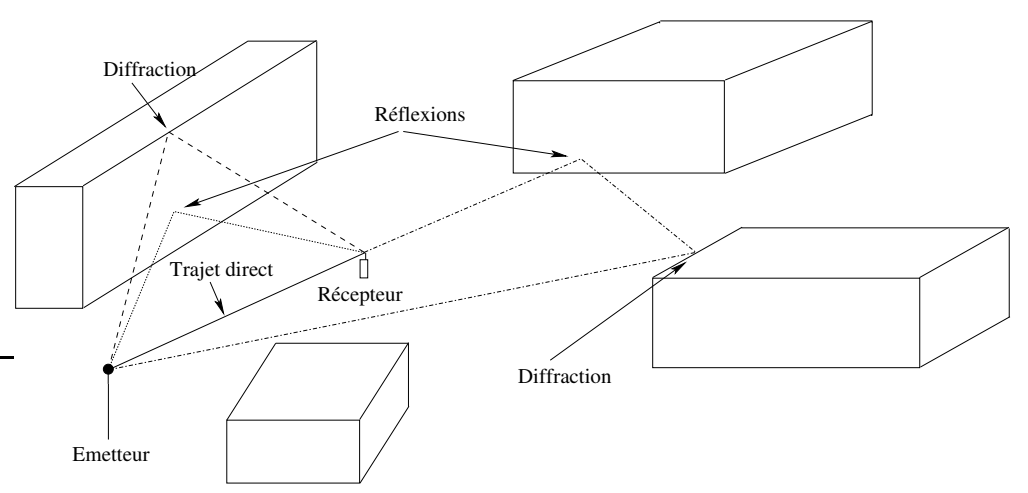

figure 19 - Propagation par trajets multiples (E : émetteur, $\mathrm{R}$ : récepteur) 


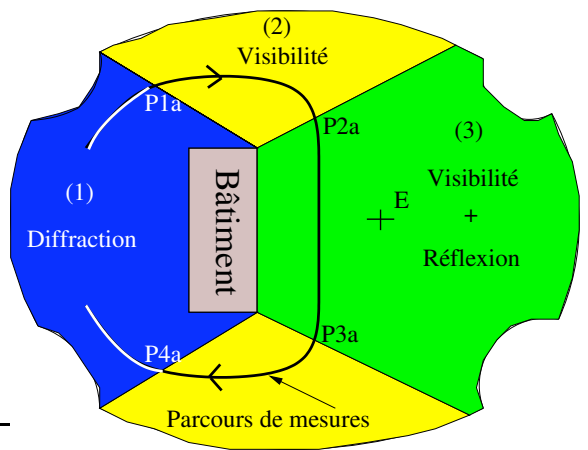

(a)

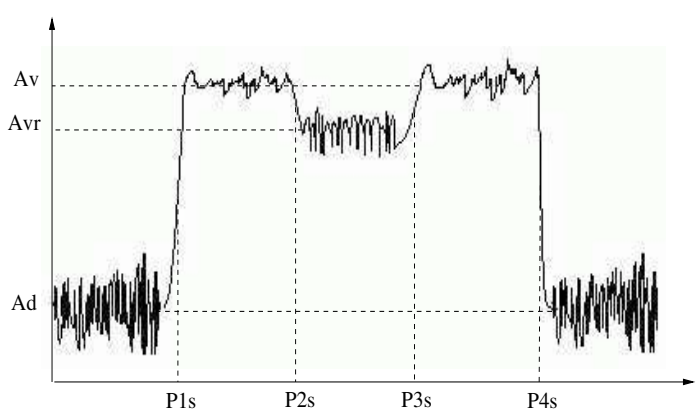

(b)

figure 20 - (a) Partition spatiale (b) Signal schématique reçu sur le parcours de mesure 


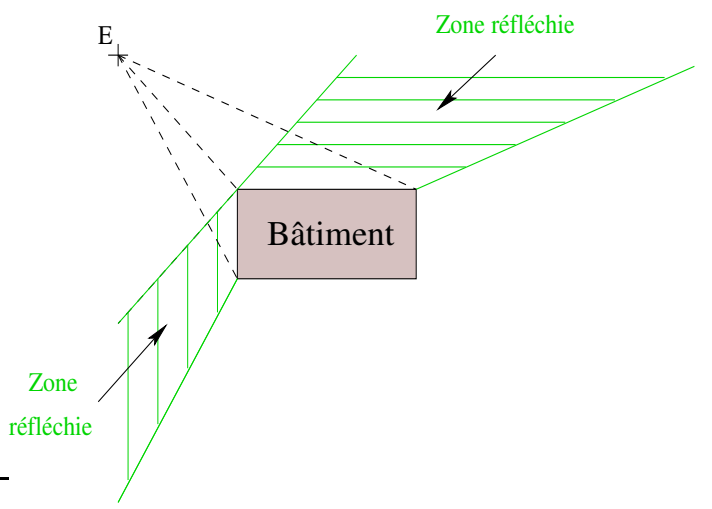

(a)

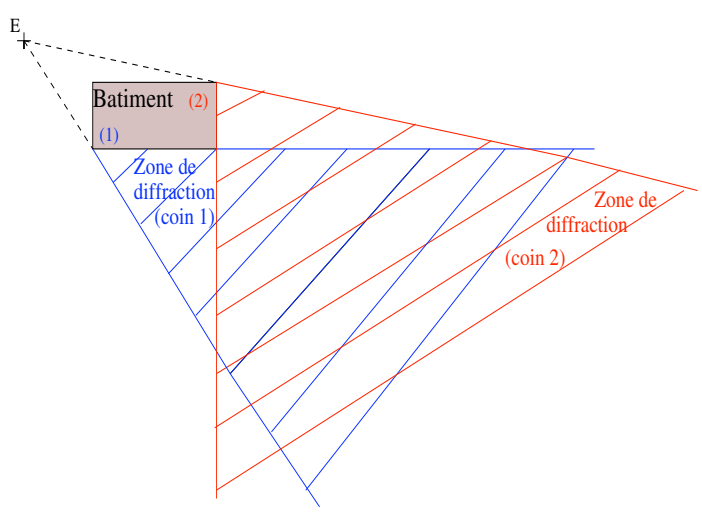

(b)

figure 21 - Zones réfléchies (a) et diffractées (b) par un bâtiment 


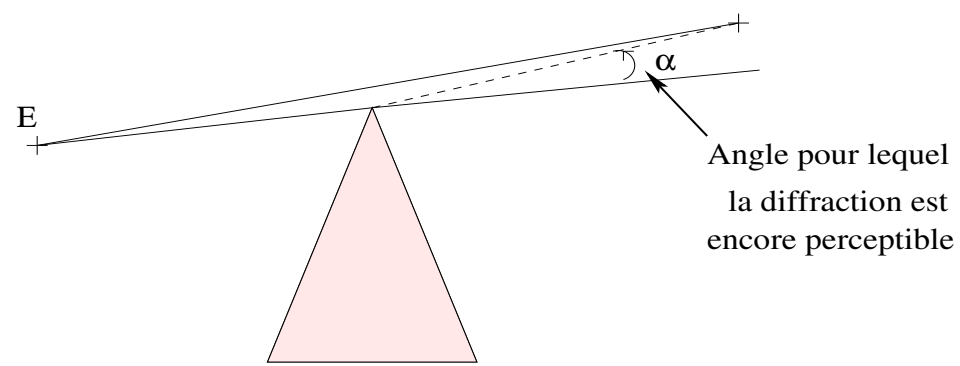

figure 22 - Limite de l'influence des ondes diffractées liée à $\alpha$ 


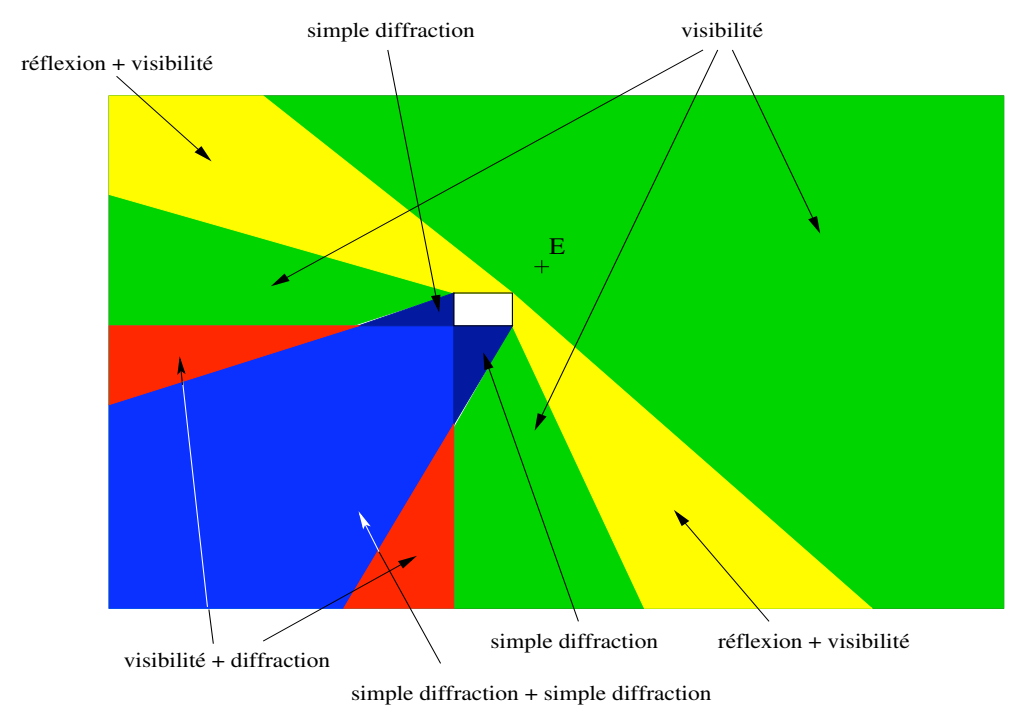

figure 23 - Les différentes zones engendrées par un bâtiment pour une diffraction et une réflexion 


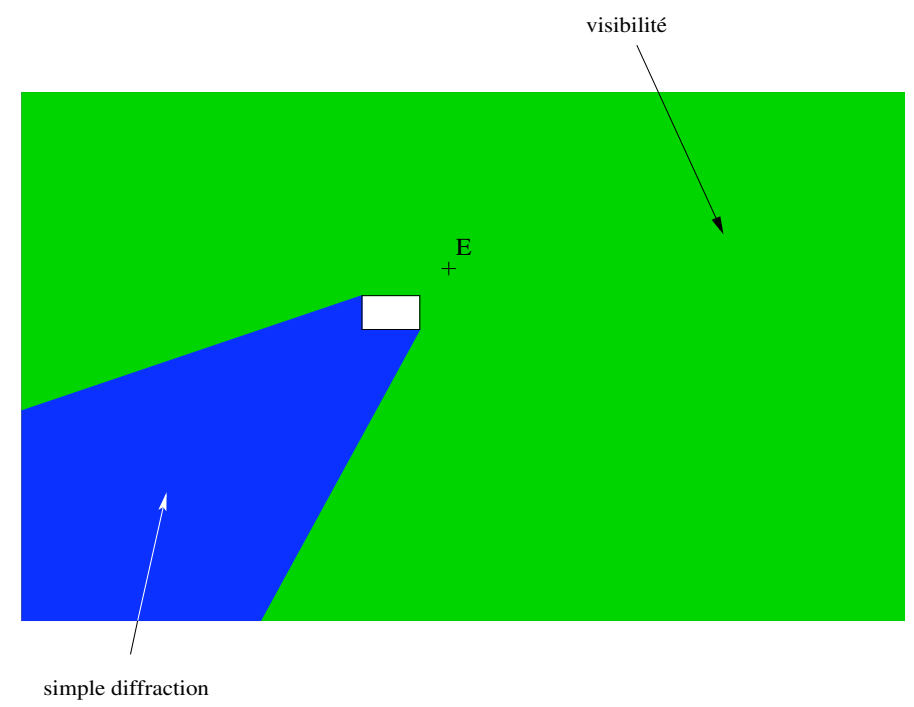

figure 24 - Résultat du regroupement 


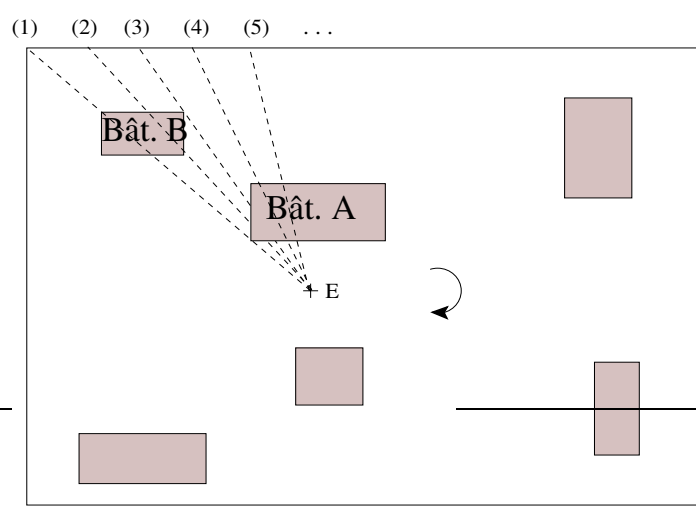

(a)

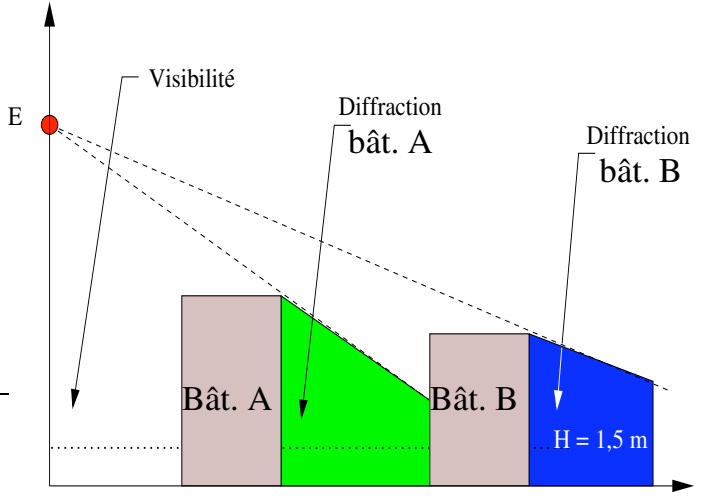

(b)

figure 25 - (a) Balayage régulier de l'environnement par des plans verticaux (b) Analyse électromagnétique du plan noté (2) pour 1 diffraction et 0 réflexion 
(1) (2) (3) (4) (5) $\quad$ (

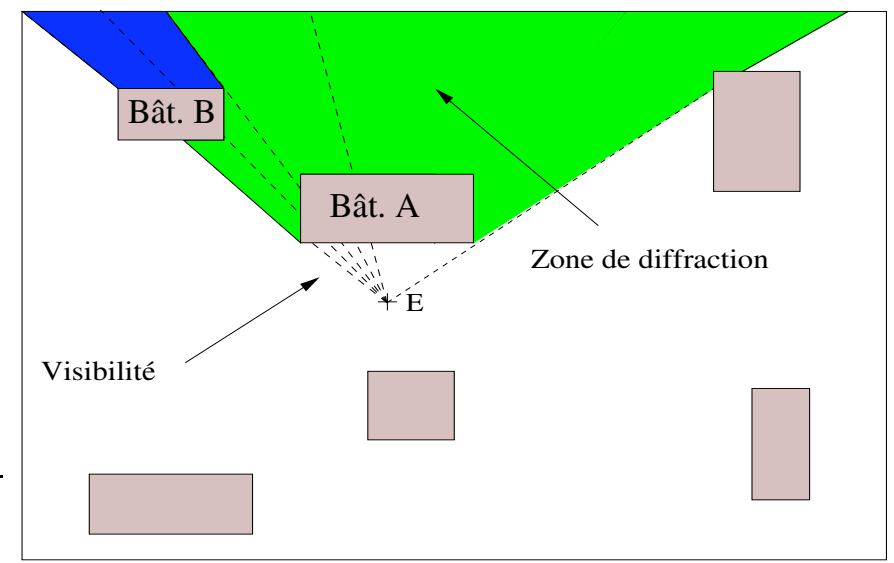

figure 26 - Construction d'une partition issue de l'analyse électromagnétique en verticale 


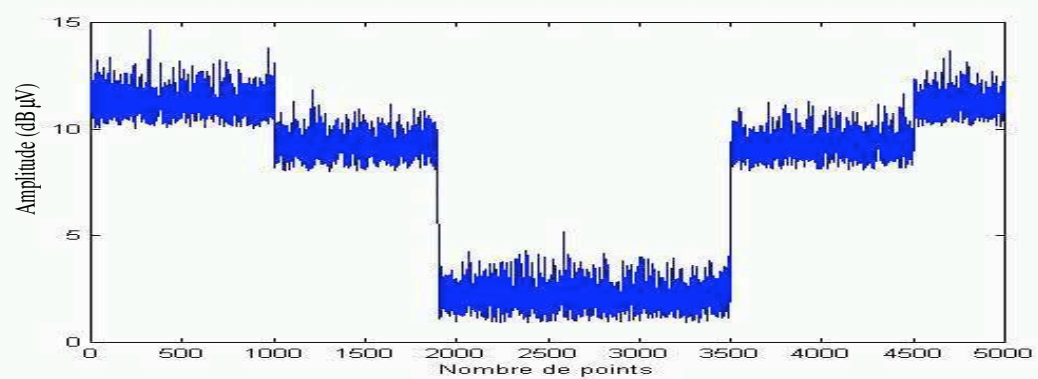

(a)

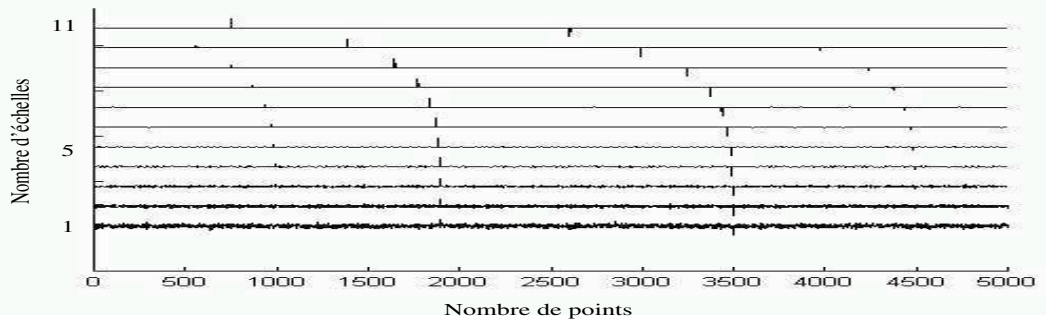

(b)

figure 27 - Segmentation d'un signal sur 11 échelles 


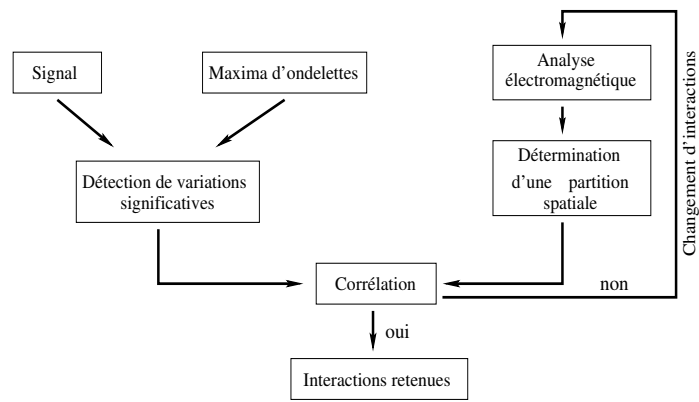

figure 28 - Organigramme de validation de l'hypothèse 


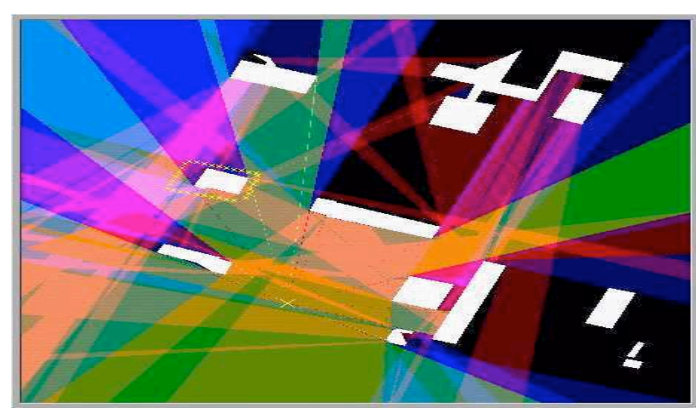

(a)

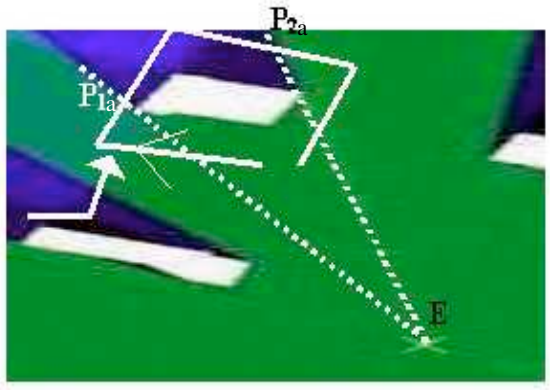

(b)

figure 29 - (a) Partition spatiale (b) Zoom sur le parcours de mesures 


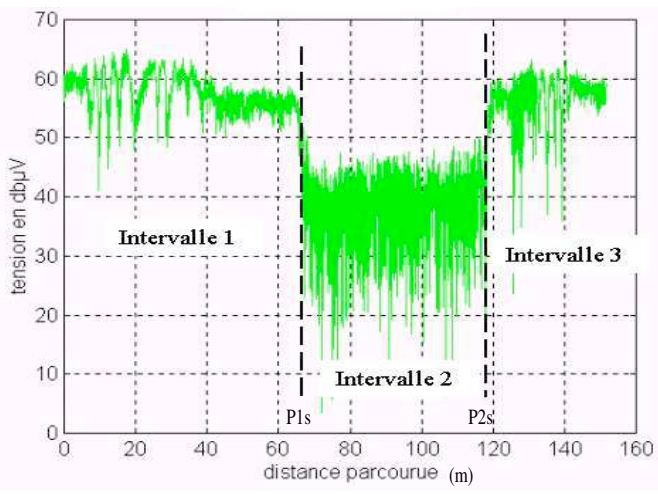

figure 30 - Signal segmenté 


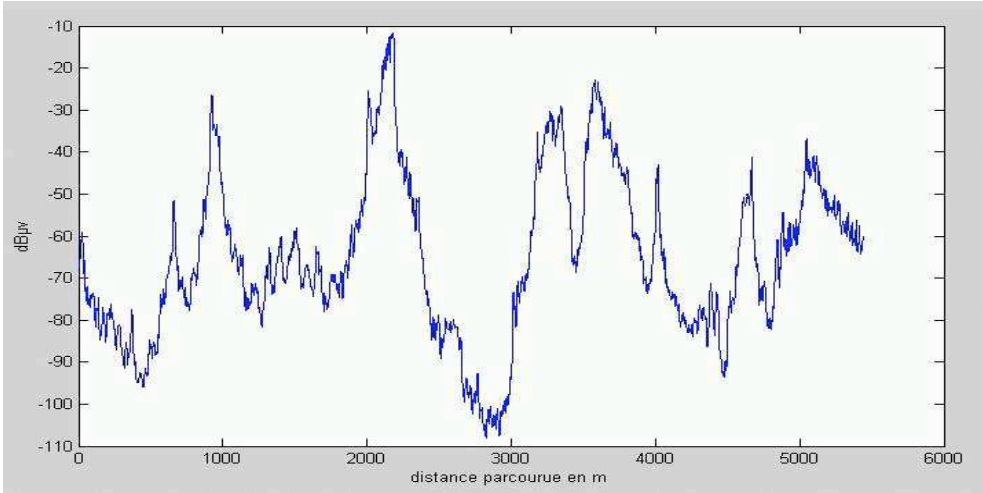

figure 31 - Signal segmenté (Paris) 


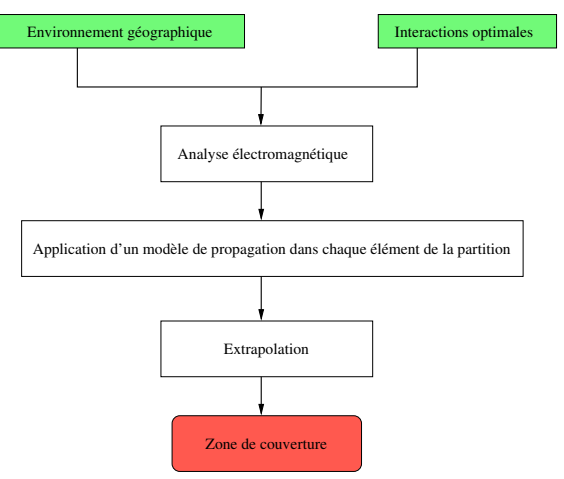

figure 32 - Organigramme de la phase d'application 


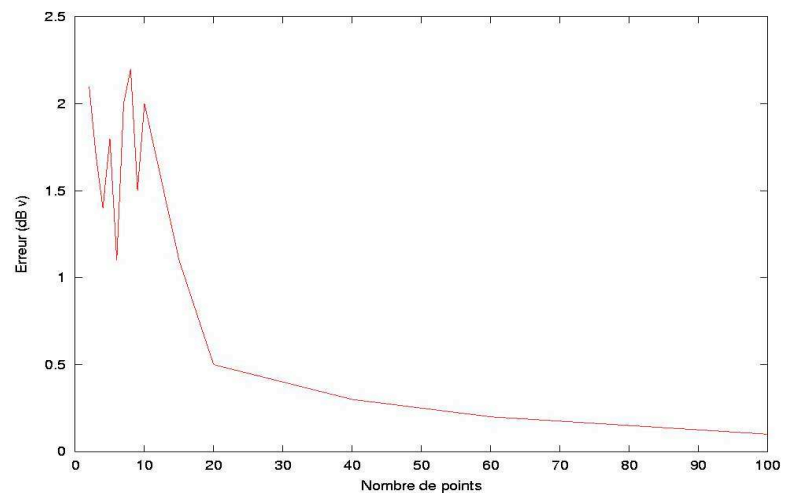

figure 33 - Evolution de l'erreur d'estimation en fonction du nombre de points considérés par segment 


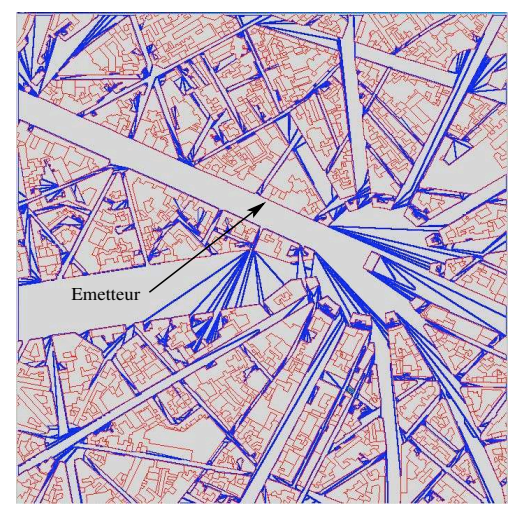

(a)

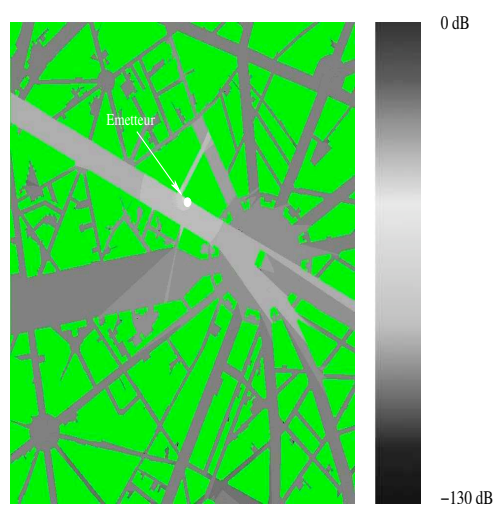

(b)

figure 34 - (a) Partition spatiale (b) Zone de couverture 


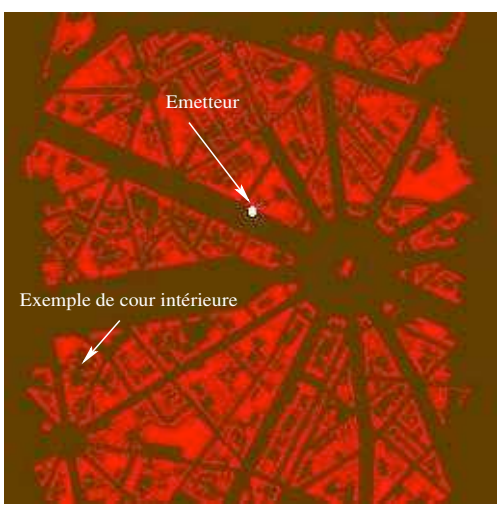

figure 35 - Vue de dessus de la scène 


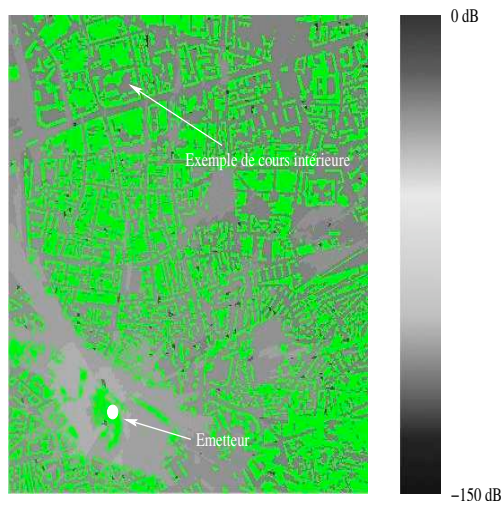

figure 36 - Visualisation de la couverture 